Res Publica Revista de Historia de las Ideas Políticas

ISSN: $1131-558 \mathrm{X}$

\title{
Sovranità e dominio nella République di Jean Bodin
}

Paolo Slongo 1

Recibido: 14-06-2020 / Aceptado: 25-02-2021

Riassunto. Questo articolo intende mettere in luce da un punto di vista storico-concettuale il problema della relazione tra Stato e sovranità nella République di Jean Bodin. Dalla seconda metà del secolo XVI e nel secolo XVII, la sovranità è intesa come un principio esterno che presuppone l'esistenza di una associazione politica. Il fine di Bodin, però, non è quello di costruire una teoria dello Stato da contrapporre alla filosofia della polis degli antichi, ma di elaborare per la prima volta una compiuta scienza politica. Viene meno così nei Six Livres de La République la netta distinzione posta da Aristotele tra la despoteia, che è relativa all'oikos, e la politeia, che è relativa alla polis. Il dominium (la despoteia) si ritrova ora sia in ambito domestico che in ambito politico. Di conseguenza accade che il potere sovrano, che pure viene equiparato al governo della casa, e che quindi dal punto di vista di Aristotele sarebbe da intendere come una forma di comando domestico, possa venir dichiarato nel caso della monarchia regia o legittima, come non dispotico. La sovranità di Bodin può dunque essere compresa come una riformulazione del dominium inteso secondo il diritto feudale.

Parole chiave: Bodin; sovranità; dominio; Stato; corpi intermedi.

\section{[es] Soberanía y dominio en La República de Jean Bodin}

Resumen. Este artículo tiene como objectivo plantear el problema de la relación entre el Estado y la soberanía en Jean Bodin. En la segunda mitad del Siglo Dieciséis y luego en el Siglo Diecisiete, la soberanía se entendió como un principio externo, que presupone la existencia de una asociación política. La terminología clásica queda entonces. Solo cuando la soberanía aparece como un elemento interno y constitutivo del cuerpo político, el término Estado tomará el lugar de la conceptualidad heredada de la tradición. Solo en esta fase específica de su desarrollo histórico-conceptual aparece el Estado como una realidad que existe en sí misma y, en consecuencia, la ciencia política se transforma en la teoría del Estado. Ciertamiente, por lo tanto, no en el pensamiento político de Jean Bodin.

Palabras clave: Bodin; soberanía; dominio; Estado; cuerpos intermedios.

\section{[en] Sovereignty and Domination in Jean Bodin La République}

Abstract. Moving from a conceptual historical perspective, the article deals with the difficult relationship among State and Sovereignty as focused in Jean Bodin's La République. Since the second half of the $16^{\text {th }}$ century and along the $17^{\text {th }}$ century, Sovereignty has always been intended as an external principle assuming the existence of some political entity. Bodin's aim is not to develop a state theory contrasting to the polis' philosophy of the ancient times, but rather to conceive for the first time a complete political science. Therefore, in the Six Livres de La République the Aristotelian clear distinction between despoteia, relating to the oikos, and politeia, relating to the polis, fails out. So, the dominium (despoteia) concerns both the domestic and the political sphere. As result it may happen that the sovereign power, being associated with the household management and therefore assuming, according to the Aristotelian definition, a character of a domestic command, in the case of a royal or legitimate monarchy could be acknowledged as non-despotic. Sovereignty in Bodin must be seen as a reformulation of the dominium of the feudal law.

Keywords: Bodin; Sovereignty; Domination; State; Intermediates Bodies.

Sommario. 1. Stato e sovranità. 2. Corpo politico e costituzione. 3. Regime e governo. 4. Signoria e dominio. 5. Potere sovrano e potere dispotico. 6. "Des Corps et Collèges, Estats, \& Communautez". Bibliografía.

Cómo citar: Slongo, P. (2021). Sovranità e dominio nella République di Jean Bodin. Res Publica. Revista de Historia de las Ideas Politicas, 24(2), 139-152.

In un saggio del 1941 intitolato Staat als ein konkreter, an eine geschichtliche Epoche gebundener Begriff (Stato come concetto concreto, legato ad un'epoca sto- rica) Carl Schmitt parla "dell'epoca della statualità". In quest'epoca, "che va dal XVI al XX secolo", lo Stato diviene "il concetto dominante di ordinamento dell'u-

1 Centro Interuniversitario di Ricerca sul Lessico Politico e Giuridico Europeo, Padova, Italia slongopaolo@gmail.com 
nità politica" ${ }^{2}$ che domina tutto. Per Schmitt, ciò che delimita l'applicabilità storica del termine Stato è la teorizzazione e l'esistenza di fatto di un potere sovrano, potere che costituisce la fonte del diritto positivo e il fondamento legittimante ogni altra autorità dello Stato. Dal momento in cui sorge lo Stato-sovrano comincia a valere, per Schmitt, una sorta di relazione biunivoca tra Stato e politica: in generale, politico viene assimilato, in una maniera o nell'altra, a statale o, quanto meno, viene riferito allo Stato ${ }^{3}$. Il punto di vista di Schmitt circa l'origine storica della sovranità è noto. Secondo Schmitt nel XVI secolo, in conseguenza della riforma protestante, si dissolve l'unità della respublica cristiana medievale e inizia la lotta tra i due grandi fronti del Weltkatholizismus e del Weltprotestantismus, cioè di un cattolicesimo con pretese universali e un protestantesimo con pretese altrettanto universali. In vista del superamento delle guerre civili confessionali, sostiene Schmitt, sorge in Francia con Jean Bodin, la concezione della decisione sovrana politica, che neutralizza tutte le altre opposizioni teologico-ecclesiastiche. La sovranità appare così come potere neutro, indipendente da questa o quella confessione religiosa: inizia la storia del moderno Stato sovrano, l'età della statualità (das Zeitalter des Staatslichkeit) che sarà destinata a durare, dice Schmitt, fino al ventesimo secolo e cioè fino al nostro presente. Presente che non è passaggio, ma "in bilico nel tempo" come dice Benjamin nelle Tesi di filosofia della storia. Schmitt ha avuto certamente il merito di delimitare l'uso storico del concetto di Stato, riferendolo alle sole formazioni politiche dell'età moderna. Egli è ben consapevole della differenza che sussiste tra quella istituzione che viene chiamata Stato moderno europeo e le realtà prestatuali, in primo luogo la società medievale, e ovviamente anche la polis classica. Per esempio, in Teologia politica, Schmitt afferma: "Nel secolo XVI sorge, dalla definitiva trasformazione dell'Europa in Stati nazionali e dalla lotta del principe assoluto coi ceti, il concetto di sovranità di Bodin"4. Ma è soprattutto in una conferenza tenuta nel 1942 nella Parigi occupata dai tedeschi, poi pubblicata su Deutschland-Frankreich ${ }^{5}$, la rivista trimestrale dello stesso Istituto Germanico di Parigi dove Schmitt l'aveva pronunciata, che egli ritorna sulla vicenda dello Stato moderno non partendo più dalla prestazione hobbesiana, come aveva fatto nel libro del $1938^{6}$, ma scoprendo

Si tratta di una parte della relazione che Schmitt tenne al Congresso Storico di Norimberga del 1941, con una postilla aggiunta per la ripubblicazione nei Verfassungsrechtliche Aufsätze aus den Jahren 1924-1954, Berlin, Duncker \& Humblot, 1958, pp. 375-385; trad. it. "Lo Stato come concetto concreto", in C. Schmitt, La formazione dell'esprit in Francia e altri scritti sull'Europa e sullo Stato, a cura di C. Pontorieri, Genova, il melangolo, 2015, pp. 103-117, qui p. 103.

3 C. Schmitt, Il concetto di "politico" (1932), in C. Schmitt, Le categorie del "politico". Saggi di teoria politica a cura di G. Miglio e P. Schiera, Bologna, il Mulino, 1972, p. 102; cf. anche C. Schmitt, "Lo Stato come concetto concreto", op. cit., p. 104.

4 C. Schmitt, Teologia politica. Quattro capitoli sulla dottrina della sovranità, in C. Schmitt, Le categorie del "politico”, op. cit., p. 43. Deutschland-Frankreich. Vierteljahresschrift des Deutschen Instituts Paris, Paris, 1, 1942, 2, pp. 1-30 (ora in C. Schmitt, Staat, Großraum, Nomos. Arbeiten aus den Jahren 1916-1969, Berlin, Duncker \& Humblot, 1995, pp. 184-210).

6 C. Schmitt, Der Leviathan in der Staatslehre der Thomas Hobbes. Sinn und Fehlschlag eines politischen Symbols, Hamburg, Han- la centralità dei giuristi nella storia politico-istituzionale francese. La sua relazione infatti si intitola significativamente: "Die Formung des französischen Geistes durch den Legisten" ". Nel suo elogio della figura, "celebre nella storia europea del diritto", del "legista" francese del secolo XVI, Schmitt scrive:

la formazione spirituale di un popolo o di una nazione nasce nella lotta (Kampf) politica reale, interna e esterna, e proviene solo da quei ceti che in tale lotta hanno assunto una posizione di guida (Führerstellung), e che, in un momento cruciale di questa lotta sono stati avanguardie e portavoce del loro popolo o della loro nazione. Come non avvenne in nessun altro popolo, il legista francese fu portavoce e battistrada nella storia dell'unità nazionale nei grandi momenti di conflitto e nella disperazione della guerra civile. Lo spirito francese è perciò spirito giuridico, e la lingua francese è per questo, più di ogni altra lingua, lingua giuridica, perché il divenire nazione della Francia ha ricevuto l'impronta decisiva proprio dal legista ${ }^{8}$.

Qualche pagina dopo, Schmitt arriva a definire la prestazione politica classica dello spirito francese: "lo Stato sovrano all'interno e verso l'esterno, che supera le contrapposizioni interne delle guerre civili di religione attraverso la decisione sovrana, cioè non mediante una decisione religiosa, ma attraverso una decisione politico-statale" . Il moderno concetto di sovranità, insomma,

è stato definito per la prima volta in Francia. Soltanto a partire da quest'epoca e soltanto tramite questa prestazione specificamente statale la Francia - dice Schmitt-è diventata patria e matrice dello spirito "classico". Un grande legista e giurista tipicamente francese, Jean Bodin (1530-1596), nel secolo della nascita dello spirito francese moderno, ha formulato la definizione del concetto di sovranità ${ }^{10}$.

Evidentemente Schmitt pensa di poter spiegare il sorgere di un concetto fondamentale del pensiero politico come quello di sovranità a partire dalla considera-

seatische Verlagsanstalt, 1938; trad. it. Il Leviatano nella dottrina dello Stato di Thomas Hobbes. Senso e fallimento di un simbolo politico, in C. Schmitt, Sul Leviatano, Introduzione di C. Galli, Bologna, il Mulino, 2011, pp. 35-128. Qui Schmitt osservava che "lo Stato, secondo Hobbes, è soltanto una guerra civile continuamente impedita da un grande potere" (ibidem, p. 56).

C. Schmitt, "La formazione dell'esprit in Francia per mezzo dei legisti", in C. Schmitt, La formazione dell'esprit in Francia, op. cit., pp. 63-102. Il testo della conferenza, che mostra il sostanziale allineamento politico di Schmitt ancora nel 1942 con il regime nazista ed è percorso da un insistente tono antisemita, si conclude emblematicamente con queste parole: "In una potente trasformazione di tutti i concetti storici, sorgono nuovi contenuti e nuove proporzioni, crescono nuovi concetti di spazio e si forma un nuovo diritto all'interno di nuovi ordinamenti. Questa volta l'ordine è conquistato dalla Germania e dall'impero. Non è però, come appare a quegli angosciati e disperati difensori della precedente misura, come se misura e diritto fossero oggi scomparsi del tutto. Ciò che finisce è solo la loro antica misura e il loro tipo di legalità. Ciò che viene è il nostro nuovo impero (Was kommt ist unser neues Reich)" (ibidem, p. 102).

Ibidem, p. 68. "L'epoca da Montaigne a Pascal è l'epoca di questi legisti «politici»" (ibidem, p. 80).

Ibidem, p. 83.

10 Ibidem, p. 84. "Il concetto decisivo dello jus publicum Europaeum, lo Stato sovrano in politica interna e in politica estera, viene da Bodin enucleato con sicurezza e avrà dopo di lui un'incomparabile fortuna. Egli è uno dei maieuti dello Stato moderno" (C. Schmitt, Ex Captivitate Salus. Esperienze degli anni 1945-47 [1950], trad. it. Milano, Adelphi, 1987, p. 68). 
zione di una specifica situazione storica, quella appunto della trasformazione dell'Europa in un insieme di Stati nazionali. Le premesse metodologiche di questo modo di argomentare sono contenute in quella che, nella terza parte di Teologia politica, Schmitt ha chiamato "sociologia dei concetti", la cui peculiarità consiste nel fatto che

viene rintracciata la struttura ultima, radicalmente sistematica, e questa struttura viene poi comparata all'elaborazione concettuale della struttura sociale di una determinata epoca. Non interessa qui se l'ideale della concettualità radicale sia il riflesso di una realtà sociologica oppure se la realtà sociale venga intesa come la conseguenza di un modo determinato di pensare e, di conseguenza, anche di comportarsi [...] Ad esempio, rientra nella sociologia del concetto di sovranità di ogni epoca mostrare che la situazione degli uomini dell'Europa occidentale nelle diverse epoche e che la conformazione giuridica della realtà politico-storica ha sempre trovato un concetto la cui struttura coincideva con la struttura dei concetti metafisici ${ }^{11}$.

In definitiva, Schmitt rimane convinto che sia necessario presupporre una certa connessione tra i concetti fondamentali della politica e una determinata "situazione" storica. Ma forse questa storia dei concetti deve liberarsi da un presupposto che è un'eredità dello storicismo, del quale Schmitt sembra restare ancora prigioniero qui: quello secondo cui la nascita del concetto di sovranità potrebbe essere spiegata in relazione ad un evento storico, la nascita dello Stato moderno europeo. Forse si dovrebbe procedere in un'altra direzione. Forse è possibile mostrare che l'affermarsi della convinzione che lo Stato sia una specifica realtà esistente nel mondo storico possa essere intesa come il momento conclusivo, l'ultima tappa delle successive modificazioni storiche che interessano le relazioni tra il concetto di sovranità e quello di Stato. L'ipotesi di una stretta connessione, di una sorta di contemporaneità storico-teorica tra il concetto di Stato e quello di sovranità non va accolta acriticamente. Innanzitutto perché è del tutto evidente come non esista contestualità, dal punto di vista del pensiero e del linguaggio politico, tra l'introduzione nella scienza politica del concetto di sovranità e l'assunzione da parte del concetto di Stato di quel ruolo di centralità che solo in seguito gli verrà attribuito. I due più grandi teorici, "che muovendo dal diritto pubblico hanno posto i fondamenti del diritto internazionale"12, Jean Bodin nel XVI secolo e poi Thomas Hobbes nel XVII secolo, non utilizzano il concetto di Stato per indicare una realtà politico-istituzionale specificamente moderna, al contrario, per essi non esiste alcuna differenza sostanziale tra la polis antica e lo Stato moderno. Si consideri quanto Hobbes afferma negli Elements of Law (Elementi di legge naturale e politica, 1640):

Questa unione così fatta è ciò che gli uomini chiamano oggigiorno corpo politico (BODY POLITIC) o società civile; e i Greci chiamano polis vale a dire città (that is to say, a city); che si può definire una moltitudine di uomini, uniti come una persona da un potere comune (united as

1 C. Schmitt, Teologia politica, op. cit., pp. 68-69.

12 C. Schmitt, Ex Captivitate Salus, op. cit., p. 66. one person by a common power), per la loro comune pace, difesa, e beneficio (for their common peace, defence, and benefit $)^{13}$.

Per porre correttamente il problema della relazione tra Stato (così è tradotto "République" nell'edizione italiana de Les six livres de la République ${ }^{14}$ ) e sovranità occorrerebbe operare una periodizzazione di quest'ultimo concetto. Nella seconda metà del Cinquecento, e poi ancora nel Seicento, la sovranità è intesa come principio esterno, presupposto all'esistenza dell'associazione politica. Permane allora la terminologia classica. Solo quando la sovranità apparirà come elemento interno, costitutivo del corpo politico, il termine Stato prenderà stabilmente il posto della concettualità ereditata dalla tradizione. Solo in questa specifica fase del suo sviluppo storico-concettuale, lo Stato appare come una realtà in sé sussistente e, di conseguenza, la scienza politica si trasforma in teoria: teoria dello Stato. Tale trasformazione però non si trova certo ancora nel pensiero politico del Cinquecento. Occorre innanzitutto osservare che, come poi Hobbes, in nessun modo Bodin teorizza una possibile distinzione tra polis e Stato moderno. La filosofia degli antichi non suscita perplessità per il fatto che essa è legata alla polis, ma perché priva di un chiaro valore scientifico. Un'avvertenza caratteristica della storia concettuale, nella cui prospettiva metodologica si muove questo nostro contributo, è quella di non scambiare il concetto con la parola e dunque non intendere il permanere nei diversi tempi della stessa parola come la testimonianza che si tratta sempre dello stesso concetto, un concetto che mostra nei diversi tempi alcune trasformazioni, anche rimarchevoli. Spesso parole antiche permangono nel moderno, ma in questo caso non si ha una trasformazione del concetto: piuttosto la vecchia parola veicola un nuovo concetto, che in genere viene a negare quel modo di pensare la politica che si racchiudeva prima nella stessa parola. Occorre ricordare qui che la differenza fondamentale tra la polis classica e lo Stato moderno, della quale deve tenere conto un'indagine di filosofia politica da un punto di vista storico-concettuale, è quella che consiste nell'assumere innanzitutto come il concetto moderno di sovranità contenga una relazione tra comando e obbedienza che è del tutto alternativa rispetto all'idea del comando politico dell'arkè politikè che caratterizza invece la concezione classica della po-

13 T. Hobbes, Elementi di legge naturale e politica, I, XIV, 8; trad. it. di A. Pacchi, Firenze, La Nuova Italia, 1985, p. 160 e nota 1 . Hobbes non ha qui evidentemente in alcun modo l'idea che esista uno Stato "moderno" diverso dalla polis classica per il fatto che nel primo, a differenza della seconda, esiste un potere sovrano, quello che egli chiama il potere comune. Piuttosto egli pensa, da teorico della politica, che un potere sovrano sia condizione universale dell'esistenza di qualsiasi corpo politico, antico o moderno che sia. Nella seconda parte degli Elements of Law, Hobbes aveva già inteso spiegare "in ultima analisi come molte persone naturali vengano unite mediante patti in un'unica persona civile, o corpo politico" (ibidem, II, I, 1, op. cit., p. 166).

14 J. Bodin, I sei libri dello Stato, a cura di M. Isnardi Parente e D. Quaglioni, 3 voll., Torino, UTET, 1964-1997, d'ora in poi indicato con la sigla Rep., il numero del libro in cifra romana e del capitolo; il numero della pagina, quando indicato, è quello di questa edizione italiana (per il testo francese facciamo riferimento a Les six livres de la République [1576], Paris, Du Puis, 1583). 
litica $^{15}$. È in Hobbes quindi che comparirà la sovranità moderna e non in Bodin, nel quale, come vedremo, la sovranità è superioritas, cioè un potere che è posto sopra una piramide e ha a che fare con una realtà complessa che non si risolve rappresentativamente in esso.

\section{Stato e sovranità}

Nell'Epistola dedicatoria della Repubblica appare lo stesso giudizio sugli antichi che, nel secolo successivo, esprimerà Hobbes nella Prefazione del suo De Cive ${ }^{16}$. Fra un milione di libri che trattano tutti i campi del sapere, dice Bodin,

se ne trovano appena tre o quattro che parlino di politica, pur essendo questa la regina delle scienze. Platone e Aristotele nei loro trattati politici ne hanno dato trattazioni così brevi che servono solo a destare il desiderio dei lettori, non certo a soddisfarli; e [...] l'esperienza ci ha portati alla convinzione che a quei tempi la scienza politica era ancora avvolta in fitte tenebre ${ }^{17}$.

L'ambizioso progetto di Bodin, di allestire con Les six livres de la Republique una scienza della politica "completa" per ampiezza e ordinata sistematicità dell'indagine, in effetti si configura come la posizione di un sapere "totale" dell'umano storico - di respiro ben più ampio della consueta "politica storica"-, un sapere poi imprescindibile da quello che riguarda il quadro naturale, quello dell' Harmonia mundi cosmologico-astrologica, nel quale l'umano è inserito, oggetto di cruciale interesse nel complesso della meditazione di Bodin. Il compito era di fornire una trattazione che non separasse economia e politica ${ }^{18}$, che investisse famiglie, corpi, collegi, ordinamenti e leggi politiche, e così via, che rendesse conto delle forme di condizionamento naturale indotte da climi e influenze celesti, che fosse infine in grado di comprendere le cause della crescita o del declinare e perire delle forme politiche. Anche il possente tentativo operato da Bodin incontrava tuttavia non poche difficoltà di ordine epistemico e metodico. Da un lato, nella costituzione di una "scienza politica empirica" bisognava abbracciare in un'indagine sistematico-enciclopedica quanti più temi e dati. Dall'altro ricompariva lo spettro del carattere indefinito della realtà empirica insieme con l'antico divieto aristotelico di una scienza del particola-

15 Sul "metodo" della storia concettuale, in particolare nell'interpretazione originale della Begriffsgeschichte sviluppata in Italia dalla scuola di Padova, cf. almeno i saggi contenuti in S. Chignola e G. Duso, Storia dei concetti e filosofia politica, Milano, FrancoAngeli, 2008. Sul punto relativo all'arkè politikè e alla sovranità moderna, cf. G. Duso, "Il potere e la nascita dei concetti politici moderni", e "La storia della filosofia politica tra storia concettuale e filosofia", ibidem, pp. 158-200 e pp. 273-296.

16 T. Hobbes, De Cive. Elementi filosofici sul cittadino, trad. it. a cura di T. Magri, Roma, Editori Riuniti, 2018, pp. 68-70.

17 Rep., pp. 135-6. La scienza politica, dice Bodin, è "una scienza talmente utile che per mezzo di essa si reggono tutti i regni e tutte le comunità regolate da norme di diritto $[. .$.$] E tuttavia è così raro tro-$ vare chi abbia scritto di essa che con grande meraviglia vediamo, in molte epoche, appena uno che abbia trattato di questa scienza" (Rep., p. 153).

18 A. Becker, "Bodin on Oeconomics and Politics", History of European Ideas, 40, 2, 2014, pp. 135-154. re: in effetti l'argomento della scienza politica "di per sé sarebbe senza fine", ammette Bodin, ricordando che "d'altronde da un pezzo, fin da fanciullo, ho appreso quella massima dei filosofi secondo la quale del particolare non si dà scienza"19.

Insomma, il fine di Bodin non è quello di costruire una teoria dello Stato da contrapporre alla filosofia della polis degli antichi, ma di elaborare per la prima volta una compiuta scienza politica. Ciò è evidente innanzitutto dal fatto che gli non utilizza per lo più il termine "stato" (status-estat) per indicare il corpo politico, ma piuttosto quello di respublica (République). La Repubblica inizia con la definizione del corpo politico (Rep., I, 1). Ricordiamo le definizioni delle due edizioni, francese e latina. Nell'edizione francese: la republique è un "droit gouvernement de plusieurs mesnages, et de cequi leur est commun, avec puissance souveraine". Nell'edizione latina: Respublica "est legitima plurium familiarum et rerum inter se communium cum summa potestate gubernatio" 20 . Per Repubblica "si intende il governo giusto che si esercita con potere sovrano su diverse famiglie e su tutto ciò che esse hanno in comune fra loro" $(I, 8)$. Bodin dimostra di essere ben consapevole di aver fornito del corpo politico una definizione diversa da quella che ne hanno dato gli antichi. Cicerone e Aristotele, egli dice, hanno chiamato repubblica una società di uomini riuniti per vivere bene e felicemente (I, 1). Ma ad una tale definizione mancano tre concetti fondamentali: quello di famiglia, quello di sovranità, e quello di beni comuni alla società nel suo insieme. Bodin ribadisce qui ciò che è già contenuto nel significato che egli attribuisce al termine repubblica, cioè il fatto che il concetto di sovranità (puissance souveraine-summa potestas) rientra nella definizione del corpo politico. La definizione di sovranità è data all'inizio del capitolo 8 del libro I: ed. fr. (1576): Souveraineté est la puissance absolue et perpetuelle d'une republique. Ed. lat. (1586): Maiestas est summa in cives ac subditos legibusque soluta potestas.

\section{Corpo politico e costituzione}

Bodin ritiene di essere stato il primo filosofo politico a definire la sovranità: "tale definizione non c'è stato mai giurista né filosofo politico che l'abbia data, e tuttavia è questo il punto più importante e più necessario a comprendersi in qualsiasi trattazione sulla repubblica" (Rep. I, 8). Le caratteristiche della sovranità sono per Bodin fondamentalmente tre: si tratta di un potere perpetuo, nel senso che non può essere limitato nel tempo. Non è possibile che il potere sovrano venga conferito per un periodo determinato scaduto il quale il sovrano non diventa altro che un suddito. Si tratta, inoltre, di un potere non limitato da alcuna legge umana (ma solo dal diritto divino): "Il principe sovrano è per legge esente dalle leggi dei predecessori, ancora meno egli sarà obbligato a osservare le leggi e le ordinanze fatte da lui

19 Rep., pp. 152-153. Cf. D. Quaglioni, La sovranità, Roma-Bari, Laterza, 2004, p. 53.

20 Rep., I, p. 159, con l'osservazione di Margherita Isnardi Parente a nota 1 . 
stesso" (Rep. I, 8). La legge, infine, dipende essa stessa dal potere sovrano: "La legge dipende da colui che ha la sovranità; egli può obbligare tutti i sudditi, e non può obbligare se stesso" (Rep. I, 8).

Bodin non pensa certamente che la sovranità inizi ad esistere dal momento in cui egli la definisce. Egli non ritiene cioè che la sua scienza politica costituisca la precondizione perché possa comparire per la prima volta nella storia un corpo politico correttamente costituito, cioè fornito di potere sovrano. Egli pensa piuttosto che sempre, in ogni formazione politica di ogni tempo, vi è stato un potere sovrano. Per cui crede di individuarne i termini corrispondenti nei linguaggi del passato. Sovranità, egli dice, è ciò che i Latini chiamavano maiestas, i Greci akrà exousia, kuria arché, kurion politeuma, gli Italiani "signoria", ecc. Ovviamente l'equiparazione che Bodin pensa di individuare tra la sovranità da lui definita e il politeuma dei Greci non ha alcun fondamento. Se facciamo riferimento al pensiero di Aristotele, è evidente che l'idea di una potestas publica che non solo non appartiene alla res publica (politeia), ma addirittura è condizione della sua esistenza, non trova alcuna corrispondenza. Occorre tener conto allora che l'introduzione da parte di Bodin del concetto di sovranità modifica nella sostanza, rispetto ad Aristotele, la relazione tra corpo politico e costituzione ${ }^{21}$.

Nella Politica (III, 6) Aristotele, dopo aver definito la politeia come ordine della polis, in particolare delle magistrature (archai), aggiunge che la politeia è, in particolare, l'ordine delle più importanti tra le magistrature, e la più importante dappertutto è il governo. Infine, conclude addirittura identificando governo e costituzione (politeia e politeuma). Ovviamente le tre affermazioni sono collegate da un nesso logico. La politeia, nel senso della cittadinanza, è per Aristotele l'insieme di coloro che, in quanto cittadini, nella loro pluralità costitutiva, godono in forma diretta e immediata del diritto di partecipazione alle cariche pubbliche, e in particolare al governo (politeuma) $)^{22}$.

Aristotele intende come kurion politeuma il governo, il quale dev'essere conforme alla costituzione; esso non è altro infatti che la suprema delle magistrature, di cui la politeia è l'organizzazione. Le leggi (nomoi), che nel loro insieme formano la costituzione, regolano l'ordine e la funzione delle cariche e indicano le modalità di partecipazione ad esse. Un comando esercitato nell'ambito della polis in modo difforme o tale da non tener conto della costituzione è simile al comando dispotico (ad esempio, nella tirannide e nella democrazia di massa o plebiscitaria, la quinta forma di democrazia nella classificazione di Pol. IV, 4). A differenza del comando

21 Cf. P. Accattino, L'anatomia della città nella Politica di Aristotele, Torino, Tirrenia Stampatori, 1986, pp. 139-146.

22 In Pol. III, 5, 1278 b 8-10, in particolare, la costituzione viene fatta coincidere con l'ordine della polis, di tutte le cariche. Aristotele la definisce come l'ordine degli abitanti di una polis (Pol. III, 1, 1274 b 38-39), anche se chiarisce subito dopo che non tutti gli abitanti sono sottoposti a questo ordine, ma solo coloro che partecipano al governo della città (Aristotele, Politica, 1275 a 19-23, a cura di C. A. Viano, testo greco a fronte, Milano, Rizzoli, 2002, p. 226). G. Bien, Die Grundlegung der politischen Philosophie bei Aristoteles, Freiburg-München, Karl Albert, 1973; trad it. La filosofia politica di Aristotele, Bologna, il Mulino, 1985, p. 304. che si esercita nelle istituzioni politiche, la despoteia è comando di uno solo, monarchia (Pol. I, 7), il quale non ha da tener conto di nessuna legge. È a questo tipo di comando che sembra corrispondere la potestas legibus soluta di Bodin.

Il rapporto che Bodin stabilisce tra il suddito (sujet-subiect-subditus) -che per essenza obbedisce e non comand-e il sovrano, che per essenza comanda soltanto e non obbedisce a nessuno (fuorché a Dio e al diritto di natura) appare quindi del tutto analogo all'arché despotiké aristotelica ${ }^{23}$, dove c'è da un lato chi comanda e mai ubbidisce, e dall'altro chi sempre ubbidisce e mai comanda ${ }^{24}$. Ciò che in Aristotele caratterizza invece l'arché politiké è il fatto che qui il cittadino (polites) è in grado tanto di comandare quanto di obbedire. Nella costituzione ottima, nell'aristocrazia, il fine dell'obbedienza è anzi soprattutto quello di apprendere a comandare. Così pure nella costituzione denominata politeia, quella alla quale corrisponde la democrazia nelle costituzioni devianti, vale il principio del comandare a turno (katà meros). La sovranità di Bodin può essere quindi intesa, in linea generale, come una trasposizione a livello pubblico-politico di una relazione che è del tutto analoga alla despoteia classica, di una relazione di comando cioè che Aristotele riteneva propria dell'ambito domestico-patrimoniale dell'oikos ${ }^{25}$. Bodin non rompe certo del

Aristotele, Pol., I, 7; III, 6, 1278 b 30-37; VII, 14, 1333 a 6. Cf. P. Accattino, op. cit., pp. 86-91.

24 Fondamentale è sottolineare come per Aristotele la città sia la più alta forma di aggregazione politica, altro sarà l'impero o lo stato nazionale. La comunità è koinonia. La prima comunità adatta alla sopravvivenza giornaliera è la famiglia, al di là del bisogno immediato, il villaggio, all'interno del quale ogni capofamiglia regna. Ma la città è una koinonia diversa, ha la qualità dell'autosufficienza, fine e natura stessa della comunità. Nell'ambito della comunità si esplica l'alternanza del comandare e dell'obbedire che riguarda la stessa ontologia dell'essere animale e poi umano: "è dunque possibile, in armonia con quanto andiamo dicendo, osservare innanzitutto nell'animale un principio di comando di carattere signorile e politico, perché l'anima esercita sul corpo un'autorità padronale, l'intelletto esercita sull'appetito un'autorità politica e regia" (Pol., I, 1254b, 2-7). L'autorità padronale e l'autorità politica, dunque, con un'intuizione estremamente feconda nel pensiero politico successivo, non coincidono. L'autorità politica si esercita su liberi per natura, non presuppone il comando di uno solo e, aggiunge Aristotele, riguarda i liberi e gli uguali.

25 "Somiglianze con le costituzioni, che, anzi, fungono quasi da modelli, si potranno trovare anche nelle comunità familiari. Infatti, la comunità che c'è tra padre e figli [25] ha la struttura di un regno, giacché il padre ha cura dei figli. E per questo che anche Omero chiama Zeus «padre»: il regno vuol essere un'autorità paterna. Tra $\mathrm{i}$ Persiani, invece, l'autorità del padre è tirannica: trattano i figli come schiavi. Tirannica, poi, è anche l'autorità del padrone nei riguardi degli schiavi: [30] in essa, infatti, si fa solo l'interesse del padrone. Ma mentre quest'ultima autorità è manifestamente corretta, quella dei Persiani, invece, è errata, giacché differenti devono essere i modi di governare uomini differenti. La comunità di marito e moglie è manifestamente di tipo aristocratico: il marito, infatti, esercita l'autorità conformemente al suo merito, e nell'ambito in cui è il marito che deve comandare; quanto invece si addice alla moglie, [35] lo lascia a lei. Il marito, invece, che comanda su tutto trasforma la comunità matrimoniale in oligarchia, perché fa questo al di là del suo merito, cioè [1161a] non per quanto è superiore alla moglie. Talvolta, poi, comandano le mogli, quando sono delle ereditiere: quindi, la loro autorità non deriva dal valore personale, ma si fonda sulla ricchezza e sul potere, proprio come nelle oligarchie. La comunità dei fratelli assomiglia a quella timocratica: essi, infatti, sono uguali, tranne che nella misura in cui [5] differiscono per età; perciò, se la differenza d'età è grande, non sorge più l'amicizia fraterna. La democrazia, infine, si trova soprattutto nelle case dove non c'è un padrone (giacché qui sono tutti su un piano di uguaglianza) e in quelle in cui chi comanda è debole e ciascuno può fare quello che vuole" (Aristotele, 
tutto con la tradizione del pensiero politico classico. Egli riprende l'idea che esistano forme di costituzione. L'espressione che Bodin usa per indicare la costituzione, la politeia, è "l'estat d'une République" (Rep. II, 2), e nella versione latina status rei publicae ${ }^{26}$. Nel 1566 intitolava l'importante capitolo sesto della sua Methodus, che è strettamente connesso con la dottrina costituzionale: De statu rerumpublicarum. In Repubblica, II, 1 egli dice che non ci sono che tre regimi o tre forme di repubblica: la monarchia, l'aristocrazia e la democrazia. Ma le intende come forme diverse di organizzazione di un potere sovrano che rimane sempre lo stesso:

monarchia si chiama lo Stato in cui uno solo ha la sovranità, e il resto del popolo ne è escluso; democrazia o regime di popolo quello in cui tutto il popolo o la sua maggioranza riunita in assemblea ha il potere sovrano (a la puissance souveraine); aristocrazia quello in cui una minoranza riunita in corpo, ha il potere sovrano (a la souveraineté en corps) e dà la legge al resto del popolo, sia in generale sia ai singoli ${ }^{27}$.

\section{Regime e governo}

Si è visto come Bodin distingua diverse forme di repubblica, cioè diversi regimi, il monarchico, l'aristocratico e il democratico. Il regime dipende da chi è detentore della sovranità. Se il sovrano è uno si tratta di una monarchia, se è un numero ristretto di un'aristocrazia, se è tutto il popolo o la sua maggioranza riunita in assemblea, di una democrazia ${ }^{28}$. A questa distinzione egli ne aggiunge un'altra, differente dalla prima, quella tra forme di governo: anche qui vale la tripartizione di monarchico, aristocratico e democratico. "Regime (estat) e governo (gouvernement), afferma Bodin, sono due cose nettamente differenti", e si tratta di una differenza che finora nessuno ha descritto ("qui est une reigle de police qui n'a point esté touchée de personne", Rep., II, 2). Per cui è possibile, ad esempio, che vi sia una repubblica monarchica con governo democratico. Questo avviene "se il principe permette a tutti di partecipare alle assemblee e agli stati, alle magistrature, agli uffici, alle ricompense, senza riguardo alla nobiltà, alle ricchezze o al merito" (Rep., II, 2). Così pure vi può essere una repubblica monarchica a governo aristocratico "se il principe non conferisce potere o benefici che ai nobili o ai più meritevoli o ai più ricchi”. Ugualmente vi può essere una repubblica aristocratica a governo democratico,

Etica Nicomachea, a cura di C. Natali, Bari-Roma, Laterza, 2010, VIII, 1160b, 25-35; 1161a, 5-10).

26 C. H. McIlwain, Costituzionalismo antico e moderno (1947), a cura di N. Matteucci, Bologna, il Mulino, 1990, p. 62, nota 10.

27 Rep., I, p. 544. Per il confronto con il testo latino, cf. A. Di Bello, Stato e sovranità nel De Republica libri sex di Jean Bodin, Prefazione di D. Quaglioni, Napoli, Liguori, 2014, p. 235. Sull'aspetto costituzionale della tripartizione delle forme politiche come "status", D. Quaglioni, I limiti della sovranità. Il pensiero di Jean Bodin nella cultura politica e giuridica dellıetà moderna, Padova, Cedam, 1992, p. 143. Cf. anche J.-F. Spitz, Bodin et la souveraineté, Paris, PUF, 1998, pp. 44-54

28 Rep. II, 1. e così via ${ }^{29}$. In sostanza, poiché tre sono i regimi, cioè le forme di repubblica, e tre i governi, si hanno nove combinazioni diverse.

È evidente come una distinzione tra forme di regime e forme di governo sia impensabile in Aristotele: la sua premessa è infatti l'introduzione del concetto di sovranità, inesistente nel pensiero politico classico. Alla distinzione delle forme di repubblica e delle forme di governo Bodin ne aggiunge un'altra, quella tra i diversi modi di esercizio del governo. Un governo, sia esso di tipo monarchico, aristocratico o democratico, può essere esercitato in modo legittimo, dispotico o tirannico. Bodin tratta questa distinzione in modo particolare in relazione al governo monarchico. La monarchia regia o legittima, che è quella in cui i sudditi obbediscono alle leggi del re e il re alle leggi di natura, restando ai sudditi la libertà naturale e la proprietà dei loro beni. La monarchia dispotica è quella in cui il principe si è fatto signore dei beni e delle persone stesse dei sudditi per diritto d'armi e di guerra giusta, "guerra giusta è quella legittimamente condotta tra repubbliche sovrane, e non quella fatta da bande di predoni o ladroni", e governa i sudditi come un capo di famiglia i suoi schiavi. "La monarchia tirannica è quella in cui il monarca calpesta le leggi di natura, abusa dei liberi come di schiavi, dispone dei beni dei sudditi come di beni propri” (Rep., II, 2). In conclusione, vi sono in Bodin tre distinzioni: quella delle forme di repubblica, quella delle forme di governo e quella tra le modalità d'esercizio del governo. Il modo di esercitare il governo è comunque cosa diversa dalla sovranità di per sé stessa considerata: il potere sovrano resta in ogni caso non contestato nella sua assolutezza. Se il principe è sovrano in assoluto, il suddito non ha alcun potere di opporglisi, neppure nel caso che questi governi in forma dispotica o addirittura tirannica: "mai un suddito può essere giustificato dell'aver tramato contro il suo principe sovrano, sia pur questo il più malvagio $\mathrm{e}$ crudele dei tiranni [...] è necessario all'occorrenza sapersi anche rassegnare alla morte piuttosto che attentare in alcun modo alla sua vita e al suo onore" (Rep., II, 5). Il suddito non ha alcun potere di sottoporre a giudizio il suo principe, "dal quale egli deriva ogni autorità e ogni potere di comando, e che può revocare ogni potere ai suoi sudditi" (Rep., II, 5). Bodin include quindi il dispotismo tra le forme di esercizio del governo in una repubblica sovrana. Si può pensare che la sovranità di Bodin possa essere intesa, in linea generale, come una sorta di trasposizione a livello pubblico-politico di una relazione che è del tutto analoga alla despoteia classica, di una relazione di comando cioè che Aristotele riteneva propria dell'ambito domestico-patrimoniale ${ }^{30}$. Aristotele

29 Th. Berns, "Bodin: la souveraineté saisie par ses marques", Bibliothèque d'humanisme et Renaissance, LXII, 3, 2000, pp. 611623; B. Périgot, "La notion de République chez Bodin", in N. Dockès-Lallement, G.-A. Pérouse, J.-M. Servet, sous la direction de, L'Euvre de Jean Bodin, Paris, Garnier, 2004, pp. 39-54; D. Marocco Stuardi, La République di Jean Bodin. Sovranità, governo, giustizia, Milano, Franco Angeli, 2006, pp. 39-50; cf. anche P. Magnard, "Jean Bodin ou l'harmonie dans la cité", Cahiers de philosophie politique et juridique de l'Université de Caen, 2, 1982, pp. 57-68.

30 L'autorità che anima la respublica "et qui peu à peu régularise son existance en lui imposant les normes du droit, peut donc se définir par analogie avec celle du paterfamilias, commis à la direction du 
distingueva infatti nettamente il governo della casa da quello della polis. Bodin nega espressamente che tale distinzione sia giustificata. In Repubblica I, 2, egli dichiara che a suo modo di vedere a torto "Aristotele e Senofonte hanno separato nettamente economia e politica"; e, più avanti dice:

per amministrazione domestica noi intendiamo il governo giusto della famiglia, che consiste nell'autorità che il capo della famiglia esercita sui suoi e sull'obbedienza che questi gli devono: e ciò non è stato affatto considerato da Aristotele e Senofonte nei loro trattati. Come la famiglia ben governata è la vera immagine della repubblica, come l'autorità domestica somiglia al potere sovrano, così il governo giusto della casa è il vero modello del governo della repubblica (Rep., I, 2).

Viene dunque meno, in Bodin, la netta distinzione posta da Aristotele tra la despoteia, che è relativa all'oikos, e la politeia, che è relativa alla polis. Il dominium (la despoteia) la si ritrova ora sia in ambito domestico che in ambito politico ${ }^{31}$.

\section{Signoria e dominio}

Si può avanzare l'ipotesi che questa trasformazione che il concetto di dominio subisce in Bodin rispetto al modo in cui era inteso nella filosofia politica classica si possa spiegare in relazione alla concezione che di questo termine era presente nel diritto feudale. Nel linguaggio dei documenti ufficiali del Medioevo dal VII fino al XII secolo il termine dominium si trova combinato con altri termini usati come suoi sinonimi, quali ad esempio ius, potestas, proprietas, possessio ${ }^{32}$. Otto Brunner ha mostrato come nel linguaggio giuridico tedesco a dominium corrisponda il concetto di Gewere. Tale termine sta ad indicare il possesso e l'utilizzo effettivi di una cosa, tali da lasciar presumere l'esistenza di un diritto vero e proprio sulla medesima ${ }^{33}$, tale possesso "presuppone, in termini giuridico-territoriali, la piena capacità difensiva" 34 . Chi è padrone di una

premier groupe naturel, la famille" (P. Mesnard, L'État de la Renaissance et son évolution vers l'absolutisme, in Encyclopédie française, t. X, L'État, Paris, Larousse, 1964, p. 18). Quella di Mesnard resta ancora una delle migliori sintesi del pensiero politico di Bodin.

31 C. Bruschi, "Pouvoir, «Mesnage» et République", in L'Évvre de Jean Bodin, op. cit., pp. 19-38. O. Beaud, La Puissance de l'État, Paris, PUF, 1994, p. 30 e p. 41.

32 Cf. alla voce "Herrschaft" (P. Moraw), in O. Brunner, W. Conze, R. Koselleck, a cura di, Geschichtliche Grundbegriffe. Historisches Lexikon zur politisch-sozialen Sprache in Deutschland, Stuttgart, Klett-Cotta, 1974, t. III, p. 9 e pp. 12-13.

33 Con questo termine si indica un istituto germanico messo in evidenza da studiosi ottocenteschi, che permetteva il legame materiale tra uomo e terra al di fuori di istituti giuridici. È traducibile, come atto, in "presa di possesso". M. Bloch, I caratteri del diritto consuetudinario, in M. Bloch, La società feudale, trad. it. Torino, Einaudi, 1999, p. 136.

34 O. Brunner, Terra e potere. Strutture pre-statuali e pre-moderne nella storia costituzionale dell'Austria medievale, Milano, Giuffrè, 1983, Introduzione di P. Schiera, p. 231 ss., 355 ss. Sull'importanza del concetto di "Gewere" nel diritto germanico, cf. E. Cortese, Le grandi linee della storia giuridica medievale, Roma, Il Cigno GG Edizioni, 2000, pp. 194-196 e p. 531. Cf. E. Conte, "Affectation, gestion, propriété. La construction des choses en droit medieval", in Aux origines des cultures juridiques européennes. Yan Thomas entre $\cos a$, di un appezzamento di terreno e di suolo, esercita legalmente la violenza nel caso la usi a tutela della propria Gewere: in altri termini, egli esercita così la sua "difesa e protezione" e dispone di un "dominium quod protectionem"35. La giurisprudenza medievale, aggiunge Brunner, intende il dominium come concetto generale, posto al di sopra dell'imperium e della potestas. Nel dominium è dunque compreso molto di più del semplice diritto di proprietà ${ }^{36}$. E ancora il signore è tale in quanto titolare di un potere (signoria) personale: egli non ha impiegati, ma servi; i suoi dominati non sono "membri" (Genosse) di associazione, ma soggetti ${ }^{37}$. Il dominium (la Gewere) trova espressione e realizzazione in quello che è l'elemento centrale, il nucleo della costituzione in senso medievale, cioè nella signoria, che trova nella casa del signore il suo nucleo fondamentale. Si tratta di una istituzione che può essere intesa, in termini generali, come lo sviluppo e l'ampliamento dell'oikos inteso in senso classico. Al signore di casa "spetta 1'esercizio della violenza legittima sulla terra; con essa egli protegge chiunque risieda nella sua casa, ovvero i congiunti, i dipendenti e l'intera familia"38. La casa costituisce l'elemento fondamentale e costitutivo della costituzione intesa in senso medievale ${ }^{39}$. Se si volesse trovare nei dizionari e nelle enciclopedie moderne un significato univoco di Herrschaft, si resterebbe frastornati dalla pluralità di significati che al termine vengono accostati. I corrispondenti in latino più o meno classico potrebbero essere: auctoritas, dignitas, ditio, dominatus, dominium, imperium, jurisdictio, majestas, potestas, principatus, territorium ${ }^{40}$. Non si può non notare che tutte queste espressioni hanno una comune connotazione autoritaria o comunque esprimono una unitarietà di dominio, di giurisdizione, di imperio, di territorio che il termine nel medioevo non ha ancora, significando relazioni di signoria-dipendenza molto diversificate, dalla signoria sulla casa e quindi anche sulle persone che vi abitano, libere o non libere che siano, a quella sulla terra e sulle persone che la coltivano ${ }^{41}$, li-

droit et sciences sociales. Études réunies par P. Napoli, Rome, École française de Rome, 2013, in part. pp. 79-81.

35 "Il potere del signore si presenta dunque come signoria, protezione, avvocazia, tutela, mundio. Il suo simbolo è la mano armata in atto protettivo" (O. Brunner, op. cit., p. 310; cf. P. Schiera, "Introduzione", ibidem, p. XXIV).

36 Infatti, il rapporto che si stabilisce fra signore e soggetto è un rapporto bilaterale, ma non nel senso del contratto moderno che vincola le persone soltanto ad un determinato risultato nel campo dei diritti di cui singolarmente possono disporre (come quello di proprietà ad esempio), bensì nel senso di un legame personale che vincola il signore alla protezione e difesa e il soggetto alla fedeltà (in cui sono compresi il consiglio e l'aiuto). O. Brunner, op. cit., p. 367.

37 M. Weber, "I tipi del potere", in M. Weber, Economia e società (1922), I, trad. it. a cura di P. Rossi, Milano, Edizioni di Comunità, 1961, pp. 207-211. È chiaro che per Weber la Herrschaft, come ben spiega Pietro Rossi nella "Nota del traduttore" apposta a M. Weber, Il metodo delle scienze storico-sociali (1922), a cura di P. Rossi, Torino, Einaudi, 1958, pp. 51-52, è il potere "nel senso di un dominio sociologicamente accertabile di un certo uomo, o di certi uomini, sul resto del gruppo sociale a cui appartiene o appartengono". "Herr" è per Weber qualsiasi titolare di potere, con una caratterizzazione più specificamente signorile nel caso del potere tradizionale.

8 O. Brunner, op. cit., p. 359.

39 E. Conte, Affectation, gestion, propriété, op. cit., pp. 80-81.

40 Geschichtliche Grundbegriffe, op. cit., alla voce "Herrschaft", p. 14.

41 E. Conte, Affectation, gestion, propriété, op. cit., p. 86. Cf. G. Vismara, "La disciplina giuridica del castello medievale (sec. VI-XIII)" 
bere o non libere che siano dal punto di vista personale. La medesima indeterminatezza si trova nei dizionari e anche nei manuali di storia del diritto quando, per spiegare la signoria su uomini di per sé liberi, si fa ricorso indifferentemente all'ambito naturale delle relazioni di età (senior - signoria - seigneurie) o a quella della casa (domus - dominus - dominatio $)^{42}$. Ciò tuttavia che evidentemente qui viene meno è l'idea del cittadino inteso come colui che, esercitando il dominio (la despoteia) nell'ambito domestico, acquisisce la facoltà di partecipare al governo politico nell'ambito della sfera pubblica, della polis, acquisisce cioè il diritto di partecipare alla politeia, alla cittadinanza. Se, da un lato, è possibile affermare che la concezione della politica che ha origine con i Greci viene del tutto meno -il concetto stesso di politica torna a essere usato nel Medioevo solo dopo la recezione dell'Etica Nicomachea e della Politica aristoteliche nel XIII secolo ${ }^{43}$ - da un altro lato si può dire che è nell'idea medievale del dominium che sono da individuare le premesse dell'origine del moderno concetto di sovranità ${ }^{44}$. La questione della sostanziale continuità che esiste tra la despoteia, intesa in senso classico, e la nozione di sovranità, si complica dunque

(1972), ora in G. Vismara, Scritti di storia giuridica, vol. 4, Milano, Giuffrè, 1988, pp. 1-133.

42 Geschichtliche Grundbegriffe, "Herrschaft", op. cit., p. 15. Anche per il paese campione dell'assolutismo europeo, la Francia, la ricerca identifica con maggior chiarezza le istituzioni per ceti dell'Ancien Régime nel loro significato regionalistico. Scrive Gerhard Oestreich: "La monarchia assoluta non era affatto in grado di annullare i tradizionali privilegi politici della società per ceti posta al suo servizio o di sopprimere gli estesi diritti di signoria locale. La lotta del diritto consuetudinario fondato sui privilegi contro ogni nuova legislazione statuale moderna e contro il principio di diritto romano di un potere legislativo appartenente esclusivamente al principe è caratteristica di tutto l'assolutismo europeo" (G. Oestreich, "Problemi di struttura dell'assolutismo europeo", in Lo stato moderno. I. Dal Medioevo all'età moderna, Testi a cura di E. Rotelli e P. Schiera, Bologna, il Mulino, 1971, p. 178). Sui limiti dell'assolutismo in Francia nel sec. XVI, cf. A. Jouanna, Le pouvoir absolu. Naissance de l'imaginaire politique de la royauté, Paris, Gallimard, 2013, pp. 130-137. Cf. F. Cosandey, R. Descimon, L'absolutisme en France. Histoire et historiographie, Paris, Seuil, 2002, pp. 40-44.

43 Geschichtliche Grundbegriffe, op. cit., t. IV, p. 802

44 Così, nello studio della forma giuridica del potere statale si comprende la preferenza accordata da Yan Thomas alla "maestà", figura forgiata secondo l'ordine della comparazione con altri livelli di estensione e di gerarchia, che si inscrive in una scala del valore istituzionale e sociale, rispetto alla sovranità, grandezza non decomponibile in parti relative. Il perno nella nozione latina di majestas ruota intorno alla misurabilità della sua grandezza, "car, d'emblée, ce trait laisse voir -scrive Thomas- ce qui la distingue de la souveraineté moderne: son aptitude à différer par le plus et par le moins. $\mathrm{Au}$ XVIème siècle, la majesté est entièrement confondue avec la souveraineté. Les publicistes de la monarchie française, et Bodin en particulier, mais également les monarchomaques, ont cru voir dans le concept romain de maiestas l'équivalent de la souveraineté, en lui prêtant la fixité requise pour la construction dont celle-ci occupe le centre: toute république, et l'ultime sujet de la puissance en chacune d'elles, monarque ou peuple, possède absolument et indivisiblement la maiestas souveraine" (Y. Thomas, "L'institution de la Majesté", Revue de Synthèse, 3-4, 1991, p. 336). Commenta Paolo Napoli: "La maestà sarebbe pertanto all'origine una nozione graduata, decomponibile secondo un ordine gerarchico, carattere che sarebbe stato riassorbito dall'unicità indecomponibile e indivisibile della sovranità moderna, nozione ormai esclusivamente qualitativa e non più quantitativa come l'antica majestas" (P. Napoli, "La sovranità mutilata. Considerazioni su un'esperienza post-foucaultiana", Scienza \& Politica, vol. XXVII, 52, 2015, pp. 67-83, qui p. 81). Con Bodin era stata avviata, scrive Paolo Napoli, "la traiettoria di una nozione di maestà che confluisce nella sovranità e finisce così per diluire la molteplicità dei suoi livelli nell'unicità e indivisibilità della grandezza" (Ibidem, p. 82). per il fatto che in Bodin permane, accanto al concetto di sovranità, l'uso del termine "dispotico", il quale assume nel giurista francese un senso del tutto diverso da quello che possedeva in Aristotele. Con esso Bodin non indica più esclusivamente il comando domestico ${ }^{45}$, ma un tipo particolare di governo della repubblica, in particolare la monarchia governata in modo dispotico, che è quella nella quale il sovrano non riconosce il suddito come legittimo proprietario. La monarchia dispotica è, per Bodin, originaria dell'Oriente, mentre non apparterrebbe alla tradizione europea. Furono gli Ungari (Bodin intende gli Unni),

popolo di stirpe affine alla tartara, con la loro venuta in Europa, a mostrare ai Germani e ai Franchi l'esempio della monarchia dispotica, proclamandosi signori di tutti i beni dei paesi conquistati [...] Solo dopo che Tedeschi, Longobardi, Franchi, Sassoni, Borgognoni, Goti, Ostrogoti, Inglesi ebbero conosciuto dagli Ungari la consuetudine asiatica cominciarono a considerarsi signori, se non delle persone, di tutte le terre dei vinti ${ }^{46}$.

Gli Ungari, devastando col ferro e col fuoco l'Europa, introdussero fra i Longobardi e gli Alemanni, popoli germanici, e fra i Franchi, antichi coloni dei Galli, l'uso di arrogarsi il dominio dispotico di tutti i beni, sì che nessuno poteva possedere una zolla di terra se non per loro beneficio. Si tratta in ogni caso di un'istituzione in rapida decadenza, dato che "le monarchie dispotiche che restano oggi sono assai poche, in confronto a tanti tiranni che vediamo sussistere". Certamente, aggiunge Bodin, essa non era tollerata dai romani, tanto che "l'imperatore Augusto, pur essendo in realtà il più gran monarca della terra, aveva in orrore l'essere chiamato signore (dominus, despotes)". Egli ammette però l'esistenza di una possibile obiezione: "Se mi si obbietta che in Europa tutti i re pretendono l'alta signoria sui beni dei sudditi, che ciascuno ammette che tutto ciò che egli possiede gli proviene dal suo proprio sovrano, rispondo che questo non basta perché riconosce il suddito vero proprietario e gli lascia la facoltà di signoria eminente $\mathrm{o}$ diretta". La signoria eminente o diretta è intesa da Bodin come un residuo del diritto germanico medievale, che, di fatto, pur continuando a sussistere, non limita la signoria dei sudditi. In ogni caso, continua Bodin, un tale tipo di signoria era sconosciuta ai Romani, "infat-

45 L'oikos, in Aristotele, non è la casa unifamiliare moderna, né semplicemente la famiglia allargata, ma un organismo complesso in cui si intrecciano rapporti eterogenei che, in Pol., 1253 b, vengono distinti in tre gruppi: relazioni "dispotiche" padrone-schiavi (che includono di solito la direzione di un'azienda agricola di ampie dimensioni), relazioni "paterne" genitori-figli, relazioni "gamiche" marito-moglie: "Il termine «capofamiglia» (despotes) -scrive Aristotele- non denota una scienza (epistemein), ma un certo modo di essere", ibid., 1255 b). Ciò che unisce queste relazioni "economiche" (di cui Aristotele sottolinea la diversità: ibid., $1259 \mathrm{a}-\mathrm{b}$ ) è quello che è stato definito un "paradigma gestionale" che "implica decisioni e disposizioni che fanno fronte a problemi ogni volta specifici, che riguardano l'ordine funzionale (taxis) delle diverse parti dell'oikos" (G. Agamben, Il Regno e la Gloria. Per una genealogia teologica dell'economia e del governo, Vicenza, Neri Pozza, 2007, p. 31). M. P. Mittica e S. Vida, "Dispotismo e politica in Aristotele", in D. Felice, a cura di, Dispotismo. Genesi e sviluppi di un concetto filosofico-politico, Napoli, Liguori, 2001, vol. I, pp. 1-32. Cf. A. Di Bello, op. cit., pp. 238-239. 46 Rep., II, 2. 
ti in tutto il diritto romano [...] non si trovano mai le parole dominium directum e dominium utile". Si tratta, quindi, di una terminologia che appartiene al diritto feudale ${ }^{47}$.

\section{Potere sovrano e potere dispotico}

Occorre qui operare una breve digressione allo scopo di chiarire l'origine e il significato di queste due ultime espressioni usate da Bodin. Nel diritto romano dominium è sinonimo di proprietas, e del più arcaico mancipium. Per esempio in Plinio: "dominium dicitur etiam proprietas, seu jus proprietatis" ${ }^{48}$. L'espressione che viene comunemente usata per designare la proprietà è dominium ex iure Quiritum, il quale consiste nella piena dominazione e disposizione su una cosa ${ }^{49}$. Non si tratta di una serie di facoltà distinte (di godere, di usare, di passare, di alienare ecc.), ma di una signoria unitaria che può essere limitata solo temporaneamente dal concorso di diritti altrui (iura in re aliena), quali ad esempio le servitù o gli usufrutti ${ }^{50}$. Dominium non viene invece usato per indicare l'autorità in ambito politico, ma si riferisce alla sola signoria privata sulle cose, opposta tanto alla patria potestas, quanto all'imperium. Anche nel caso della signoria sugli schiavi -che dal punto di vista giuridico sono "cose", ma da quello naturale sono pur sempre delle persone- il diritto romano evita regolarmente la dizione di dominium. Sotto questo aspetto occorre riconoscere l'esistenza di una differenza tra il dominium dei Romani e la despoteia dei Greci, in quanto, come si è visto, a differenza del primo quest'ultima si applica anche nei confronti delle persone, cioè ai servi e agli schiavi. Comunque sia, l'uso del termine dominium -quale concetto generale sotto il quale ricadano tanto il

47 A. Gouron, "La coutume en France au Moyen Age", in Recueils de la société Jean Bodin pour l'histoire comparative des institutions, 52, 2, La coutume, Bruxelles, 1990, pp. 193-217. Per Tommaso d'Aquino, ad esempio, c'erano solo due tipi di dominium esistenti in natura, "il dominium utile, cioè il diritto naturale, o potere, di usare ciò di cui si ha bisogno per sostenere la propria vita, e il dominium di un superiore su un inferiore per il bene di quest'ultimo. Quest'ultimo è un dovere imprescindibile, che deriva da una superiorità naturale, $\mathrm{e}$ non un «diritto». Per analogia, l'Aquinate include in questo stesso caso il dominium politico, con il quale egli intendeva la iurisdictio direttiva e di governo, i doveri dei giusti governanti che mirano al bene dei loro sudditi (Summa Theologiae Ia IIa 94.5). È in ogni caso possibile ritrovare nell'Aquinate [...] l'idea che si possa avere la proprietà di un'altra persona, cioè di uno schiavo. Secondo Tommaso, questo dominium directum di un signore su di uno schiavo, o di un proprietario sulla sua terra, non era prescritto dalla legge naturale, ma era contemplato dalle leggi umane" (J. Coleman, "«Proprietà»: premoderna e moderna", in S. Chignola e G. Duso, a cura di, Sui concetti giuridici e politici della costituzione dell'Europa, Milano, FrancoAngeli, 2005, pp. 127-128).

48 Plinio, Hist. Nat. 8, 37. Cf. L. Capogrossi Colognesi, "Proprietà (diritto romano)", in Enciclopedia del Diritto, Milano, Giuffrè, vol. XXXVII, 1988, pp. 160-225, qui pp. 219-220.

49 Cf. S. Romeo, L'appartenenza e l'alienazione in diritto romano: tra giurisprudenza e prassi, Milano, Giuffrè, 2010, pp. 57-61. Y. Thomas, Il valore delle cose (2002), a cura di M. Spanò, con un saggio di G. Agamben, Macerata, Quodlibet, 2015, pp. 74-75 e Y. Thomas, "Res, choses et patrimoine. Note sur le rapport sujet-objet en droit romain", Archives de Philosophie du Droit, 25, 1980, pp. 413-426.

50 P. Grossi, "Dominium, dominium rei, dominium iuris", in P. Grossi, Il dominio e le cose. Percezioni medievali e moderne dei diritti reali, Milano, Giuffrè, 1992, pp. 111-118; e P. Grossi, "La categoria del dominio utile e gli Homines novi nel quadrivio cinquecentesco", ibidem, pp. 247-280. potere quanto le signorie di ogni specie è, in questa sede, qualcosa di totalmente sconosciuto. Troviamo tuttavia nel diritto romano (nel Digesto) la distinzione tra l'actio directa, in cui consiste il diritto del proprietario di cedere l'oggetto, dall'actio utilis, che è ogni altro diritto diverso da quello ${ }^{51}$. Chi, ad esempio, gode di un diritto di usufrutto non è abilitato all' actio directa. Si tratta di una distinzione che, in forma generale, può venir ricondotta a quella tra proprietà e possesso. Poiché nel Medioevo si perde il senso unitario del concetto di dominium del diritto romano ${ }^{52}$ è possibile determinare in esso una distinzione ricalcata su quelle che nel diritto romano erano actiones: la distinzione tra actio directa e actio utilis viene a trasformarsi in quella di dominium directum e dominium utile $^{53}$. Vale la pena di richiamare il punto della questione. Cioè come le origini del moderno concetto di potere, in Bodin, possano essere ricercate nella despoteia classica. Poiché la sociologia politica intende la politica sostanzialmente come uso del potere, si può affermare che con tale equiparazione vada radicalmente perduto il significato della politeia classica, intesa come la forma di comando che uomini liberi esercitano su altri uomini ugualmente liberi, e quindi essenzialmente distinta dal comando dispotico. Se il termine sovranità, dal punto di vista storico concettuale, costituisce il momento di passaggio dalla despoteia dei classici al potere dei moderni, tale passaggio si potrà dire compiuto però solo nel momento in cui, nella fondazione del concetto di sovranità ${ }^{54}$, verrà meno ogni distinzione tra il comando

$51 \quad$ R. Feenstra, "Dominium utile est chimaera? Nouvelles réflexions sur le concept de propriété dans le droit savant (à propos d'un ouvrage récent)", TRG, 66, 1998, pp. 381-397; E. Cortese, "Controversie medievali sul dominio utile. Bartolo e il «quidam doctor de Aurelianus»", in Amicitiae Pignus. Studi in ricordo di Adriano Cavanna, Milano, Giuffrè, 2003, I, pp. 613-635.

52 P. Grossi, "La proprietà e le proprietà nell'officina dello storico", in P. Grossi, Il dominio e le cose, op. cit., pp. 603-665 e "«Dominia» e «servitutes». (Invenzioni sistematiche del diritto comune in tema di servitù)", ibidem, pp. 57-122; cf. anche P. Grossi, "Proprietà (diritto intermedio)", in Enciclopedia del Diritto, XXXVII, Milano, Giuffrè, 1988, pp. 226-254, qui p. 228.

53 La distinzione di dominium directum e dominium utile risale al giurista Pillio da Medicina, che insegna a Modena negli ultimi anni del secolo XII e muore a Padova dopo il 1207. Si tratta di una teoria di grande portata che favorisce l'innesto del diritto feudale nel diritto romano. Infatti, mentre Bologna è concentrata esclusivamente sullo studio del diritto giustinianeo, Pillio a Modena allarga i suoi orizzonti assumendo tra le materie d'insegnamento i Libri feudorum, una raccolta di consuetudini feudali lombarde. Pillio elabora infatti, a proposito del feudo, la distinzione tra la nuda proprietà (il dominio diretto) che appartiene al signore, al detentore di una signoria, e un forte diritto reale su cosa altrui (il dominio utile). Il nome di diritto utile deriva dalle 'azioni utili' che il superficiario (il titolare di un diritto di superficie), l'enfiteuta (colui che gode di un diritto reale su un fondo altrui) e il conduttore di lungo termine possono esercitare. Su tutto questo cf. E. Conte, "Modena 1182, the origins of a new paradigm of ownership. The interface between historical contingency and the scholarly invention of legal categories", Glossae: European Journal of Legal History, 15, 2018, pp. 4-18. L'espressione dominium eminens si ritroverà ancora addirittura in Grozio, il quale intende con esso il potere che appartiene allo Stato di limitare in vario modo i diritti e le libertà dell'individuo (J. Coleman, "«Proprietà»", op. cit., p. 130).

54 'La souveraineté est ou n'est pas: la présupposition même de ce qui l'affecterait quantitativement est absurde et contraire à la logique de la construction étatique, comme l'a si bien vu Kelsen" (Y. Thomas, "L'institution de la Majesté", op. cit., p. 336). Kelsen si oppone decisamente alla tesi secondo cui la sovranità sarebbe una categoria storica: "Bisogna prendere posizione e sottolineare con forza che il concetto di sovranità cui la dottrina del diritto e dello Stato deve pervenire $[. .$.$] ha validità tanto per lo Stato e il diritto degli antichi Egizi$ 
dispotico e il comando politico. È solo con Hobbes che tale processo si compie realmente e pienamente. Hobbes non riconoscerà più alcuna sostanziale differenza tra il diritto di proprietà (sui beni e sulle cose in genere), il dominio su individui (schiavi o servi) e il comando politico (dominio su cittadini). Nel Leviatano (II, 20), Hobbes nega espressamente che esista una qualche differenza sostanziale tra la sovranità e il dominio paterno o dispotico ${ }^{55}$.

Le cose stanno diversamente in Bodin, che invece distingue ancora tra potere sovrano e potere dispotico. Si è detto che il governo dispotico è quello che non riconosce il suddito come vero proprietario, che non gli attribuisce il dominium utile. Bodin aggiunge che le monarchie dispotiche non appartengono alla tradizione dell'Europa, nella quale esistono monarchie regie piuttosto, o legittime, oppure, nel caso di una prevaricazione dei diritti di proprietà, monarchie tiranniche. Il governo dispotico si trova, invece, ancora in Germania e nei paesi del settentrione d'Europa, oltre che naturalmente nei paesi dell'Asia, e viene evidentemente inteso da Bodin come un residuo del diritto germanico medievale, risalente alle invasioni degli Unni. Si tratta di un governo che comporta una limitazione alla facoltà del suddito di disporre liberamente dei suoi beni. Bodin non ritiene tale situazione superata de jure, ma solo de facto. Egli non sembra quindi fornire un nuovo fondamento al diritto di proprietà. Comunque, Bodin afferma chiaramente che la distinzione tra regio e legittimo da un lato e dispotico dall'altro riguarda esclusivamente il modo di governare, solo quindi l'esercizio del potere sovrano e non ha nulla a che vedere con la sovranità in quanto tale, che è sempre e in ogni caso assoluta. Di conseguenza accade che il potere sovrano, che pure viene equiparato al governo della casa, e che quindi dal punto di vista di Aristotele sarebbe da intendere come una forma di comando domestico, possa venir dichiarato, nel caso della monarchia regia o legittima, come non dispotico. La sovranità di Bodin può dunque essere compresa come una riformulazione del dominium inteso secondo il diritto feudale. Del resto, si è visto che per Bodin maiestas e dominium (potestas), souveraineté e seigneurie, sono cose niente affatto diverse in via di principio: la sovranità poi è solo suprema potestas, che si distingue dalle autorità minori, ad essa subordinate, per il solo fatto di non ammettere alcun potere superiore. Tuttavia, esiste in Bodin una novità rispetto al diritto feudale che dipende dalla connessione che si determina tra il concetto di sovranità $\mathrm{e}$ quello di cittadino ${ }^{56}$. Certamente per Bodin, a differenza

quanto dei moderni Europei” (H. Kelsen, Il problema della sovranità e la teoria del diritto internazionale [1920], trad. it. Milano, Giuffrè, 1989, p. 10).

55 Th. Hobbes, Leviatano, trad. it. a cura di A. Pacchi, Roma-Bari, Laterza, 2004, p. 170.

56 "La storia costituzionale è di solito la registrazione di una serie di oscillazioni. Talvolta sono i diritti privati, che si pongono come il problema principale, tal'altra volta è la necessità di prevenire un disordine, che minaccia di mutarsi in anarchia, che passa innanzi a tutto: in generale il Cinquecento è segnato piuttosto da questa seconda caratteristica" (C. H. McIlwain, op. cit., p. 158). Sin dagli esordi della storia costituzionale europea il problema che si è posto è stato quello dell'imbrigliamento e della reciproca neutralizzazione, in un sistema di rapporti volti all'equilibrio, dei vettori in cui si scompone il fatto di dominio. La costituzione è stata il mezzo in cui bilanciare di Aristotele, il "cittadino" non è colui che, quando esce dall'ambito della casa dove esercita il comando dispotico, si trova come uomo libero, a partecipare al governo politico. Il cittadino resta, anche fuori dell'ambito domestico, sottoposto al dominio del sovrano ${ }^{57}$. Si legge nella Repubblica:

Quando però il capo della famiglia esce dall'ambito della casa in cui comanda per trattare e negoziare coi capi delle altre famiglie di ciò che riguarda l'interesse comune, si spoglia del titolo di padrone, capo e signore per divenire semplice compagno ed uguale agli altri, e membro della loro società, e invece che signore, all'atto di lasciare la famiglia per entrare nella città e gli affari domestici per trattare i pubblici, comincia a chiamarsi cittadino, parola che in termini precisi significa suddito (subiect) libero che dipende dalla sovranità altrui (Rep. I, 6).

In connessione con l'introduzione del concetto di sovranità si determina allora l'apertura di uno spazio pubblico-politico che trascende lo spazio domestico, l'ambito della casa, e quindi si pongono le condizioni per il superamento del dominium nel senso del diritto feudale.

\section{6. “Des Corps et Collèges, Estats, \& Communautez"}

Si è detto che la relazione tra i concetti di Stato e di sovranità va periodizzata. Finché la sovranità è intesa come principio esterno, presupposto dell'esistenza dell'associazione politica, permane ancora la terminologia classica (non viene teorizzata alcuna distinzione tra la polis classica, la civitas medievale e lo Stato moderno). Solo quando la sovranità apparirà come elemento interno, costitutivo del corpo politico, il termine Stato prenderà stabilmente il posto della concettualità ereditata dalla tradizione. Sarà in questa specifica fase del suo sviluppo storico-concettuale che lo Stato apparirà come una realtà in sé sussistente, e di conseguenza la scienza politica si trasformerà in teoria: teoria dello Stato. Ciò non avviene con Bodin. Il quale, pur avendo introdotto il termine sovranità nel linguaggio politico, per indicare il corpo politico usa il termine Respublica (République) e non quello di Stato. Quest'ultima espressione è, tuttavia, presente in Bodin, che la usa in almeno quattro diversi significati. Innanzitutto, Bodin indica con l'espressione

quella "oscillazione": "Mais il y a bien defference entre droit et loy: l'un n'emporte rien que l'equité, la loy emporte commandement: car la loy n'est autre chose que le commandement du souverain, usant de sa puissance" (Rep., I, 8). Bodin fa sua quella che era stata una delle certezze più profonde del diritto medievale, e cioè che il diritto è realtà ben diversa dalla legge del re.

57 È in questo orizzonte che occorre situare una precoce presa di distanza dal discorso medievale della cittadinanza, un discorso centrato, per un verso, sulla visione di un ordine "verticale" e gerarchico e, per un altro verso, sulla celebrazione del vincolo di appartenenza dell'individuo alla civitas come corpo politico di cui l'individuo è parte. Secondo Pietro Costa, "l'annuncio del distacco può vedersi in filigrana già in Bodin, che continua a parlare di comunità e di corpi, ma vede ormai nella gerarchia e soprattutto nel suo vertice, il sovrano, la struttura portante dell'ordine" (P. Costa, "La cittadinanza: un "geschichtlicher Grundbegriff»?" in S. Chignola e G. Duso, Sui concetti giuridici e politici della costituzione dell'Europa, op. cit., p. 259). 
status innanzitutto la forma della repubblica (estat de la République, status reipublicae), cioè la costituzione. Dopo aver parlato della sovranità e dei suoi diritti e delle sue prerogative (marques) essenziali, Bodin afferma che occorre osservare chi nelle varie forme di repubblica abbia in mano il potere sovrano, allo scopo di dare definizione dei diversi regimi (estat). Si parla di estat monarchico quando la sovranità è nelle mani di uno solo (stato del principe), di estat aristocratico se partecipa alla sovranità una minoranza del popolo; di estat polulaire (democrazia) se tutto il popolo partecipa alla sovranità (Rep. II, 1). In secondo luogo, status indica per Bodin la dignità o la carica. Nella Repubblica si parla, ad esempio, de "les estats de Maresciaux de France", o de "l'estat de Capitaine". "Faire l'estat de" significa assumere e sostenere una funzione, una carica pubblica, un ufficio: per esempio, "i re dei tempi eroici faisans l'estat de juges, de capitaines, de sacrificateurs" (Rep. II, 3 ). In questo secondo senso estat significa la condizione che permette l'esercizio del governo, anche l'esercizio stesso della sovranità. Essere sovrani si dice "possedere uno stato": "le prince souverain venu à l'estat par voye d'election, ou par sort, ou par droit successif" (II, 5). In terzo luogo, per stato si intende il pubblico dominio. È il significato usato prevalentemente da Machiavelli, di cui, com'è noto, Bodin era attento lettore ${ }^{58}$ : "Gouverner son estat avec puissance souveraine" significa disporre con potere sovrano di un insieme formato concretamente di sudditi, territori, entità sociali e giuridiche minori. È questo forse il senso che appare più vicino a quello moderno di Stato. Infine, status indica il ceto, l'assemblea di ordini: "Les estats d'un pays" sono gli ordini e i collegi, le assemblee locali, le riunioni separate dei ceti della respublica (II, 7). Si tratta di forme di associazione intermedie tra la famiglia e la repubblica.

Esse costituiscono quell'ordinamento politico che verrà denominato società per ceti, una istituzione che tra la fine del Medioevo e la prima età moderna succede al sistema feudale, quindi in sostanza alla signoria, e precede il moderno Stato di diritto ${ }^{59}$. In effetti, in Repubblica III, 7, Bodin distingue, oltre alla famiglia, che chiama comunità naturale, e alla repubblica, che è una comunità governata con potere sovrano, i collegi che egli chiama comunità civili. Per collegio, dice Bodin, s'intende l'associazione legittima di tre o più persone della stessa condizione. Un collegio può essere di un mestiere, di una scienza, di un commercio, di una giurisdizione. Ciascun collegio può avere diversi regolamenti, statuti, privilegi particolari. Esistono due tipi di collegi: quelli che si autogovernano, ma senza ovviamente trasgredire le leggi del sovrano, corpi privati; e quelli istituiti dal sovrano

58 T. Berns, Souveraineté, droit et gouvernamentalité. Lectures du politique moderne à partir de Bodin, Clamécy, Léo Scheer, 2005, pp. 121-128. Cf. S. Goyard-Fabre, Jean Bodin et le droit de la république, Paris, PUF, 1989, pp. 223-278.

59 O. Brunner, "I diritti di libertà nell'antica società per ceti", in $\mathrm{O}$. Brunner, Per una nuova storia costituzionale e sociale, trad. it. a cura di P. Schiera, Milano, Vita e Pensiero, 2000, pp. 201-216. Sulla nozione di "stato" nel Medioevo, E. Conte, "L'Etat au Moyen Age: le charme résistant d'un questionnement dépassé", in P. Bonin, P. Brunet et $\mathrm{S}$. Kerneis, sous la direction de, Formes et doctrines de l'État. Dialogue entre histoire et théorie du droit, Paris, Pedone, 2018, pp. 123-136. stesso con giurisdizione e potere di comando, persone pubbliche. Questi ultimi sono, a loro volta, di due tipi: quelli istituiti per l'amministrazione delle cose divine e non di quelle umane (per esempio i pontefici e i vescovi), e quelli costituiti da ufficiali e commissari ${ }^{60}$. L'ufficiale è definito come una persona pubblica che ha una carica ordinaria definita con un editto (legge del sovrano). Un editto o una legge è condizione essenziale per l'istituzione di un pubblico ufficio. Gli ufficiali detengono l'autorità pubblica solo nella misura in cui accettano le leggi che emanano dal monarca; quelli principali sono nominati direttamente dal sovrano, mentre gli ufficiali di rango inferiore possono anche essere designati da corporazioni e collegi, ma in ogni caso al sovrano resta il potere di confermarli al loro posto. Ovviamente il potere pubblico legittimo, che appartiene ai magistrati superiori, non deve in nessun caso essere confuso col potere pubblico sovrano che appartiene unicamente al principe. Collocata al cuore del libro di Bodin, la questione del capitolo 7 del libro III, intitolato "Des Corps et Collèges, Estats, \& Communautez", è cruciale nella letteratura politica della fine del XVI secolo ${ }^{61}$ : si tratta di definire la potenza, i privilegi, i limiti, e la necessità dei corpi intermedi collocati tra la famiglia e la république (Rep. III, 7). Questi corpi intermedi sono ancora abbastanza presenti nella Francia di Bodin da porre la questione della loro necessità, per l'esistenza di quella sostanza comunitaria $^{62}$ che essi con-dividono con la république stessa. La sovranità di questa è già sufficientemente messa in rilievo perché la questione dei corpi intermedi sia posta innanzitutto come problema, come ciò rispetto a cui si tratta di distinguere. Contrariamente alla famiglia, infatti, i collegi sono delle comunità civili, proprio come la Repubblica. Ma contrariamente a quest'ultima essi non sono governati dalla puissance souveraine: "Tous corps et colleges sont instituez, pour la religion, ou pour la police", e, quanto a questa finalità, essi "sont establis pour distribuer la justice, ou departir les charges, ou donner ordre aux provisions et marchandises qu'il faut apporter, ou enlever: ou pour les mestiers necessaires à la Republique: ou pour les mestiers necessaires à la Republique: ou pour l'institution et discipline"; essi possono dunque essere specifici di un mestiere, o di un sapere, o di una merce o giurisdizione ${ }^{63}$, essi possono combinarsi tra loro

60 A differenza dell'ufficiale, il commissario è definito come una persona pubblica che riceve una carica, un incarico straordinario limitato per editto. Si tratta di un incarico assegnato non per legge ma per mandato diretto, quindi di un potere limitato nel tempo, che per la finalità specifica che è chiamato ad attuare è dipendente e revocabile in ogni momento dal sovrano. Sui commissari in Bodin, C. Schmitt, La dittatura. Dalle origini dell'idea moderna di sovranità alla lotta di classe proletaria (1921), Bari, Laterza, 1975, pp. 36-51. Cf. T. Berns, Souveraineté, droit et gouvernamentalité, op. cit., pp. 93-97.

61 Basti pensare alla Politica methodice digesta atque exemplis sacris et profanis illustrata di Althusius (I ed. 1603, Aalen, Scientia Verlag, 1981). G. Duso, "Introduzione: Patto sociale e forma politica", in G. Duso, a cura di, Il contratto sociale nella filosofia politica moderna, Bologna, il Mulino, 1987, su Althusius in part. pp. 13-21.

62 Scrive Paolo Grossi: "l'universo politico-giuridico medievale ci si presenta quale universo di relazioni fra entità diverse racchiuse in un ordine, ma che nell'ordine serbano le loro diversità segnate dai confini relativi dell'autonomia" (P. Grossi, "Dalla società di società alla insularità dello Stato: fra Medioevo ed età moderna", in S. Chignola e G. Duso, Sui concetti giuridici, op. cit., p. 109).

63 "In questo universo ordinato dal diritto la strutturazione gerarchica si origina in un contesto oggettivo di situazioni, nasce dalle cose, 
in molti modi, e inoltre presuppongono il permesso del sovrano. Infine, essi si organizzano naturalmente intorno a qualcosa di comune, ma non in una comunità totale (Rep. III, 7). La sovranità aspira certo a presentarsi, in Bodin, come l'elemento proprio e caratteristico della comunità politica, come "il vero fondamento, il cardine su cui poggia tutta la struttura della repubblica, e da cui dipendono i magistrati, le leggi, le ordinanze: essa è il solo legame e la sola unione che fa di famiglie, corpi, collegi, privati, un unico corpo perfetto, che è appunto la repubblica"64. Tuttavia, Bodin propone una genesi pre-statuale di questi "corps et collèges", di queste parti plurali ${ }^{65}$ la cui unione fa l'unico corpo perfetto della république. In origine, essi erano detti in Grecia $\varphi \imath \lambda i ́ \tau 1 \alpha$, dice Bodin, "per l'amicizia giurata che avevano fra loro" mentre in Italia "erano chiamati sodalitia, per l'unione, il continuo stare insieme, l'amicizia che essi avevano tra loro, bevendo e mangiando insieme la maggior parte del tempo"66. Essi esprimevano così mondi vitali che si sono mantenuti in libertà e autonomia, in "ce qui leur est com-

perché è nelle cose che ognuno trova il suo posto e il suo ruolo" (P. Grossi, "Dalla società di società alla insularità dello Stato", S. Chignola e G. Duso, op. cit., p. 109). Cf. P. Grossi, Mitologie giuridiche della modernità, Milano, Giuffrè, 2001, pp. 65-66.

${ }_{64}$ Rep., I, 2, p. 177, corsivo nostro. Cf. M.-D. Couzinet, "La logique divine dans les Six livres de la République de Jean Bodin”, in Y. C. Zarka, a cura di, Jean Bodin. Nature, histoire droit et politique, Paris, PUF, 1996, p. 67.

65 "Con la cittadinanza vengono conservate nel corpo della repubblica tutte le differenze naturali, quelle che appartengono ai sudditi in quanto nati in un certo luogo o membri di un certo lignaggio, ceto o corpo sociale, e inoltre i membri di ciascuna cittadinanza si differenziano anche per tutti quei diritti di corpo e di collegio che spettano loro in quanto abitanti di una certa città. All'interno di una repubblica possono perciò darsi molteplici cittadinanze, ciascuna dotata di leggi e consuetudini differenti" (M. Scattola, op. cit., p. 64). Sulla funzione dei "corps" e dei "Colleges" che conferiscono alla società carattere pluralistico ed organico: Rep., III, cap. 7. Se è vero che la sovranità è un rafforzarsi del potere in senso strumentale e al tempo stesso ne è una relativizzazione perché lo immerge nella storia, è anche vero che, benché assoluta, "la sovranità di Bodin ha limiti: sia nelle leggi di Dio e della natura, sia nelle leggi fondamentali del regno, cioè nelle famiglie, nella proprietà privata, nella costituzione cetuale del Paese che ne costituisce l'ossatura. Una sovranità che cancellasse questa realtà sarebbe demonica, essa stessa disordinata nemica di Dio, della natura e della storia" (C. Galli, Sovranità, Roma-Bari, Laterza, 2019, pp. 76-77). Il pluriversum delle differenze reali non è mai, come ha sempre sostenuto la grande tradizione costituzionalistica a cui anche Bodin appartiene, totalmente riducibile ad Unum. M. Fioravanti, Costituzionalismo. Percorsi della storia e tendenze attuali, Roma-Bari, Laterza, 2009, p. 113.

66 "In tal modo - egli prosegue- le società e la comunità di vita mantenevano viva l'amicizia, quella fiamma sacra che rivelò in origine il suo primo ardore fra il marito e la moglie, poi fra genitori e i figli, e poi fra $\mathrm{i}$ fratelli, indi fra questi e i parenti più prossimi, e da questi infine fra gli amici” (Rep., III, 7, p. 249). Quelle comunità di vita si giustificano proprio per il fatto che mantengono l'amicizia che è il fondamento stesso della république, "e tale amicizia non si può mantenere altro che con alleanze, associazioni, stati, comunità, corporazioni, corpi e collegi". Sopprimere tutte queste "parti" del corpo politico equivarrebbe, per Bodin, a credere che "lo Stato possa reggersi senza amicizia, quell'amicizia senza di cui neppure il mondo può sussistere" (Rep., pp. 278-279). In Hobbes non resterà più alcuna traccia di questa "amicizia" che si ripartisce in tutte le comunità: il soggetto di diritto emerge per dis-sociare radicalmente le comunità, che non sono più intese se non in nome della concorrenza che esse esprimono nei confronti della sovranità, e sono definitivamente considerate come realtà secondarie i cui limiti vengono drasticamente segnati. Il rifiuto hobbesiano delle comunità intermedie si affermerà come elemento centrale e motore della definizione della sovranità, e non soltanto come una sua conseguenza. Nel pensiero politico moderno il "contratto" diviene la figura neutralizzante quando si tratta di pensare l'associazione "in un corpo". mun", per tutto il tempo in cui la giustizia sovrana è stata inesistente, o non abbastanza efficace: "L'unione fra questi ha mantenuto più popoli senza forma di Stato né di potere sovrano". Certo, i diritti, i limiti e le possibilità di questi corpi sono ormai considerati in relazione alla sovranità perpetua e assoluta, e il sovrano circoscrive la legittimità delle comunità ${ }^{67}$. Inscritto nella république, ogni corpo o collegio è un diritto di comunità legittima sottostante al potere sovrano ${ }^{68}$. Bodin precisa: "è ormai chiaro che il punto più alto della maestà sovrana e potestà assoluta (majesté souveraine et puissance absolue) sta nel dar legge ai sudditi in generale e in particolare, senza il bisogno del loro consenso" (Rep., I, 7, p. 374). E aggiunge: "Toute la force des lois gît en ceux qui ont le commandement, soit le Prince souverain, soit le Magistrat, c'est-à-dire puissance de contraindre les sujets d'obéir, ou de les punir". In questo modo, tuttavia, Bodin non fa altro che meglio mettere in risalto, per contrasto, proprio quella realtà di cui sono testimonianza la pluralità delle consuetudini, dei modi di vita comuni, per mezzo della loro specifica maniera di instaurazione e di regolazione, del loro diritto, cioè della loro singolare temporalità normativa:

67 Riportando una serie di riferimenti storici ai governanti dell'antica Grecia, della Roma repubblicana e imperiale, nonché del periodo medievale e moderno, Bodin precisa che l'aggettivo "perpetuel$l e "$ significa "per tutta la vita di colui che ha il potere", mentre "absolue" si riferisce all'assenza per il sovrano di "altri limiti che i comandi della legge di Dio e della natura" (Rep., p. 351, 354). Pertanto, "chi è sovrano [...] non deve essere in alcun modo soggetto al comando altrui, e deve poter dare la legge ai sudditi, e scancellare o annullare le parole inutili in essa per sostituirne altre, cosa che non può fare chi è soggetto alle leggi o a persone che esercitino il potere su di lui. Per questo la legge dice che il principe non è soggetto all'autorità delle leggi" (Rep., pp. 358-359). In realtà, un altro limite alla sovranità era rappresentato da "quelle leggi che riguardano la struttura stessa del regno e il suo stesso assetto fondamentale, in quanto esse sono connesse alla corona e a questa inscindibilmente unite (tale è, per esempio, la legge salica); qualunque cosa un principe faccia in proposito, il successore è in pieno diritto di abolire tutto ciò che sia stato compiuto con pregiudizio di quelle leggi su cui la stessa maestà sovrana poggia e si fonda" (Rep., p. 368).

68 Ibidem, p. 251. La potestà di "fare le leggi" è certo considerata da Bodin la principale manifestazione del potere sovrano, al punto da ricomprendere anche tutte le altre prerogative di governo (Rep., p. 495). In questo modo la potestà legislativa risulta essere inscindibilmente legata alla sovranità, al punto che tutti gli attributi della prima si riverberano automaticamente sulla seconda. In particolare, sotto il profilo della titolarità, la potestà legislativa poteva spettare solo all'entità sovrana, che non poteva dividerla con altri soggetti, in quanto "la sovranità [...] giocata a due" sarebbe stata una "assurdità grande, e del tutto incompatibile con la sovranità, e contraria sia alle leggi sia alla ragione naturale" (Rep., p. 379). Ma l'articolazione per ceti della Francia, la sua pluralità istituzionale storicamente esistente non è da Bodin negata, le comunità cittadine rimangono, già date, nella loro molteplicità "naturale". Anche se esse stanno perdendo una buona parte del loro originario significato costituzionale (A. Biral, "Jean Bodin e la moderna scienza politica", in A. Biral, Storia e critica della filosofia politica moderna, a cura di G. Duso, Milano, FrancoAngeli, 1999, pp. 43-64), la République è ancora intesa come un complesso di corpi, un plurale che il sovrano stesso deve presupporre. Specie se la nave della République e il suo pilota, il kybernētes, corre un grave pericolo: "depuis que l'orage impétueux a tourmenté le vaisseau de notre République avec telle violence, que le Patron même et les Pilotes sont comme las et recrus d'un travail continuel, il faut bien que les passagers y prêtent la main, qui aux voiles, qui aux cordages, qui à l'ancre $[. .$.$] puisque tous ensemble courent un même danger"$ (Rep., Préface). 
La coutume prend sa force peu à peu, et par longues années, d'un commun consentement de tous, ou de la plupart. Mais la loi sort en un moment, et prend sa vigueur de celui qui a puissance de commander à tous. La coutume se coule doucement et sans force. La loi est commandée et publiée par puissance, et bien souvent contre le gré des sujets (Rep., I, 10) ${ }^{69}$.

\section{Bibliografia}

Accattino P., L'anatomia della città nella Politica di Aristotele, Torino, Tirrenia Stampatori, 1986.

Agamben, G., Il Regno e la Gloria. Per una genealogia teologica dell'economia e del governo, Vicenza, Neri Pozza, 2007.

Althusius, J., Politica methodice digesta atque exemplis sacris et profanis illustrata (I ed. 1603), Aalen, Scientia Verlag, 1981.

Aristotele, Politica, a cura di C. A. Viano, testo greco a fronte, Milano, Rizzoli, 2002.

-, Etica Nicomachea, a cura di C. Natali, testo greco a fronte, Bari-Roma, Laterza, 2010.

Beaud, O., La Puissance de l'État, Paris, PUF, 1994.

Becker, A., "Bodin on Oeconomics and Politics", History of European Ideas, 40, 2, 2014, pp. 135-154.

Berns, T., "Bodin: la souveraineté saisie par ses marques", Bibliothèque d'humanisme et Renaissance, LXII, 3, 2000, pp. 611 -623.

-, Souveraineté, droit et gouvernamentalité. Lectures du politique moderne à partir de Bodin, Clamécy, Léo Scheer, 2005.

Bien, G., Die Grundlegung der politischen Philosophie bei Aristoteles, Freiburg-München, Karl Albert, 1973; trad. it. La filosofia politica di Aristotele, Bologna, il Mulino, 1985.

Biral, A., "Jean Bodin e la moderna scienza politica", in A. Biral, Storia e critica della filosofia politica moderna, a cura di G. Duso, Milano, FrancoAngeli, 1999, pp. 43-64.

Bloch, M., I caratteri del diritto consuetudinario, in M. Bloch, La società feudale, trad. it. Torino, Einaudi, 1999.

Bodin, J., I sei libri dello Stato, a cura di M. Isnardi Parente e D. Quaglioni, 3 voll., Torino, UTET, 1964-1997.

-, Les six livres de la République [1576], Paris, Du Puis, 1583.

Brunner, O., Terra e potere. Strutture pre-statuali e pre-moderne nella storia costituzionale dell'Austria medievale, Milano, Giuffrè, 1983.

-, "I diritti di libertà nell'antica società per ceti”, in O. Brunner, Per una nuova storia costituzionale e sociale, trad. it. a cura di P. Schiera, Milano, Vita e Pensiero, 2000, pp. 201-216.

Bruschi, C., "Pouvoir, «Mesnage» et République", in N. Dockès-Lallement, G.-A. Pérouse, J.-M. Servet, sous la direction de, L'Euvre de Jean Bodin, op. cit., pp. 19-38.

Capogrossi Colognesi, L., "Proprietà (diritto romano)”, in Enciclopedia del Diritto, Milano, Giuffrè, vol. XXXVII, 1988, pp. 160225.

Chignola S. e Duso G., Storia dei concetti e filosofia politica, Milano, FrancoAngeli, 2008.

Coleman, J., “«Proprietà»: premoderna e moderna”, in Chignola S. e Duso, G., a cura di, Sui concetti giuridici e politici della costituzione dell'Europa, Milano, FrancoAngeli, 2005, pp. 119-158.

Conte, E., "Affectation, gestion, propriété. La construction des choses en droit medieval”, in Aux origines des cultures juridiques européennes. Yan Thomas entre droit et sciences sociales. Études réunies par P. Napoli, Rome, École française de Rome, 2013 ,

Conte, E., "Modena 1182, the origins of a new paradigm of ownership. The interface between historical contingency and the scholarly invention of legal categories", Glossae: European Journal of Legal History, 15, 2018, pp. 4-18.

-, “L'Etat au Moyen Age: le charme résistant d'un questionnement dépassé”, in P. Bonin, P. Brunet et S. Kerneis, sous la direction de, Formes et doctrines de l'État. Dialogue entre histoire et théorie du droit, Paris, Pedone, 2018, pp. 123-136.

Cortese, E., Le grandi linee della storia giuridica medievale, Roma, Il Cigno GG Edizioni, 2000.

-, "Controversie medievali sul dominio utile. Bartolo e il «quidam doctor de Aurelianus»", in Amicitiae Pignus. Studi in ricordo di Adriano Cavanna, Milano, Giuffrè, 2003, I, pp. 613-635.

Cosandey F., Descimon R., L'absolutisme en France. Histoire et historiographie, Paris, Seuil, 2002.

Costa, P., "La cittadinanza: un "geschichtlicher Grundbegriff»?" in S. Chignola e G. Duso, a cura di, Sui concetti giuridici e politici della costituzione dell'Europa, op. cit., pp. 251-262.

Di Bello, A., Stato e sovranità nel De Republica libri sex di Jean Bodin, Prefazione di D. Quaglioni, Napoli, Liguori, 2014.

Dockès-Lallement, N., Pérouse, G.-A., Servet, J.-M., sous la direction de, L'Euvre de Jean Bodin, Paris, Garnier, 2004.

Duso, G., "Introduzione: Patto sociale e forma politica", in G. Duso, a cura di, Il contratto sociale nella filosofia politica moderna, Bologna, il Mulino, 1987, pp. 7-49.

-, "Il potere e la nascita dei concetti politici moderni", in S. Chignola e G. Duso, Storia dei concetti e filosofia politica, op. cit., pp. $158-200$.

Feenstra, R., "Dominium utile est chimaera? Nouvelles réflexions sur le concept de propriété dans le droit savant (à propos d'un ouvrage récent)", TRG, 66, 1998, pp. 381-397.

Fioravanti, M., Costituzionalismo. Percorsi della storia e tendenze attuali, Roma-Bari, Laterza, 2009.

Galli, C., Sovranità, Roma-Bari, Laterza, 2019.

Goyard-Fabre, S., Jean Bodin et le droit de la république, Paris, PUF, 1989.

69 Corsivo nostro. Bodin non fa che citare qui un assioma comune a tutti i giuristi coutumiers del suo tempo. Si riconosceva che una norma era passata "en coustume" quando questa aveva trovato applicazione per almeno quarant'anni senza provocare reclami, prova questa del tacito consentement degli interessati. La forza della coutume è nella sua temporalità, cioè in un mondo della vita che in questa esprime giuridicamente la sua normatività e istituzionalizza il tempo delle comunità. La république realizza il suo fine solo attraverso la mediazione di un corpus di leggi, editti e ordonnances che, posti e definiti dalla puissance souveraine, si aggiungono alle leggi naturali divine, alle leggi fondamentali, che sono la costituzione coutumière del regno, e alle leggi morali non scritte. Cf. V. Piano Mortari, Diritto romano e diritto nazionale in Francia nel secolo XVI, Milano, Giuffrè, 1962 , pp. 88-94. Ci permettiamo di rinviare su questo punto al nostro La forza della consuetudine. Costumi, costituzione, governo in Montaigne e Montesquieu, Milano, FrancoAngeli, 2020, pp. 21-40. 
Gouron, A., "La coutume en France au Moyen Age", in Recueils de la société Jean Bodin pour l'histoire comparative des institutions, 52, 2, La coutume, Bruxelles, 1990, pp. 193-217.

Grossi, P., "Dominium, dominium rei, dominium iuris", in P. Grossi, Il dominio e le cose. Percezioni medievali e moderne dei diritti reali, Milano, Giuffrè, 1992, pp. 111-118.

-, "La categoria del dominio utile e gli Homines novi nel quadrivio cinquecentesco", Il dominio e le cose, op. cit., pp. $247-280$.

-, "La proprietà e le proprietà nell'officina dello storico", in P. Grossi, Il dominio e le cose, op. cit., pp. 603-665

-, “«Dominia» e «servitutes». (Invenzioni sistematiche del diritto comune in tema di servitù)", Il dominio e le cose, op. cit., pp. 57-122.

-, "Proprietà (diritto intermedio)", in Enciclopedia del Diritto, XXXVII, Milano, Giuffrè, 1988, pp. 226-254.

-, "Dalla società di società alla insularità dello Stato: fra Medioevo ed età moderna", in S. Chignola e G. Duso, Sui concetti giuridici e politici della costituzione dell'Europa, op. cit., pp. 103-117.

-, Mitologie giuridiche della modernità, Milano, Giuffrè, 2001.

Hobbes, T., Elementi di legge naturale e politica, trad. it. di A. Pacchi, Firenze, La Nuova Italia, 1985.

-, De Cive. Elementi filosofici sul cittadino, trad. it. a cura di T. Magri, Roma, Editori Riuniti, 2018.

-, Leviatano, trad. it. a cura di A. Pacchi, Roma-Bari, Laterza, 2004.

Jouanna, A., Le pouvoir absolu. Naissance de l'imaginaire politique de la royauté, Paris, Gallimard, 2013.

Kelsen, H., Il problema della sovranità e la teoria del diritto internazionale (1920), trad. it. Milano, Giuffrè, 1989.

Magnard, P., "Jean Bodin ou l'harmonie dans la cité", Cahiers de philosophie politique et juridique de l'Université de Caen, 2 , 1982, pp. 57-68.

Marocco Stuardi, D., La République di Jean Bodin. Sovranità, governo, giustizia, Milano, Franco Angeli, 2006.

McIlwain, C. H., Costituzionalismo antico e moderno (1947), a cura di N. Matteucci, Bologna, il Mulino, 1990

Mesnard, P., L'État de la Renaissance et son évolution vers l'absolutisme, in Encyclopédie française, t. X, L'État, Paris, Larousse, 1964.

Mittica, M. P. e Vida, S., "Dispotismo e politica in Aristotele", in D. Felice, a cura di, Dispotismo. Genesi e sviluppi di un concetto filosofico-politico, Napoli, Liguori, 2001.

Moraw, P., voce "Herrschaft [dominium]", in O. Brunner, W. Conze, R. Koselleck, a cura di, Geschichtliche Grundbegriffe. Historisches Lexikon zur politisch-sozialen Sprache in Deutschland, Stuttgart, Klett-Cotta, 1974, t. III, pp. 5-13.

Napoli, P., "La sovranità mutilata. Considerazioni su un'esperienza post-foucaultiana", Scienza \& Politica, vol. XXVII, 52, 2015, pp. 67-83.

Oestreich, G., "Problemi di struttura dell'assolutismo europeo", in Lo stato moderno. I. Dal Medioevo all'età moderna, Testi a cura di E. Rotelli e P. Schiera, Bologna, il Mulino, 1971, pp. 173-191.

Plinio, Storia Naturale, Vol. II, Antropologia e zoologia, Libri 7-11, Torino, Einaudi, 1983.

Périgot, B., "La notion de République chez Bodin", in N. Dockès-Lallement, G.-A. Pérouse, J.-M. Servet, sous la direction de, L'Euvre de Jean Bodin, Paris, Garnier, 2004, pp. 39-54.

Piano Mortari, V., Diritto romano e diritto nazionale in Francia nel secolo XVI, Milano, Giuffrè, 1962.

Quaglioni, D., La sovranità, Roma-Bari, Laterza, 2004.

-, I limiti della sovranità. Il pensiero di Jean Bodin nella cultura politica e giuridica dell'età moderna, Padova, Cedam, 1992.

Romeo, S., L'appartenenza e l'alienazione in diritto romano: tra giurisprudenza e prassi, Milano, Giuffrè, 2010, pp. 57-61.

Schmitt, C., Teologia politica. Quattro capitoli sulla dottrina della sovranità (1922), in C. Schmitt, Le categorie del "politico". Saggi di teoria politica a cura di G. Miglio e P. Schiera, Bologna, il Mulino, 1972, pp. 29-86.

-, Il concetto di "politico" (1932), in Le categorie del "politico", op. cit., pp. 87-165.

-, "Die Formung des französischen Geistes durch den Legisten", Deutschland-Frankreich. Vierteljahresschrift des Deutschen Instituts Paris, Paris, 1942, 2, pp. 1-30, ora in C. Schmitt, Staat, Großraum, Nomos. Arbeiten aus den Jahren 1916-1969, Berlin, Duncker \& Humblot, 1995, pp. 184-217; trad. it. "La formazione dell'esprit in Francia per mezzo dei legisti", in C. Schmitt, La formazione dell'esprit in Francia e altri scritti sull'Europa e sullo Stato, a cura di C. Pontorieri, Genova, il melangolo, 2015, pp. 63-102.

-, "Staat als ein konkreter, an eine geschichtliche Epoche gebundener Begriff" (1941), in C. Schmitt, Verfassungsrechtliche Aufsätze aus den Jahren 1924-1954, Berlin, Duncker \& Humblot, 1958, pp. 375-385; trad. it. "Lo Stato come concetto concreto", in C. Schmitt, La formazione dell'esprit in Francia, op. cit., pp. 103-117.

-, Der Leviathan in der Staatslehre der Thomas Hobbes. Sinn und Fehlschlag eines politischen Symbols, Hamburg, Hanseatische Verlagsanstalt, 1938; trad. it. Il Leviatano nella dottrina dello Stato di Thomas Hobbes. Senso e fallimento di un simbolo politico, in C. Schmitt, Sul Leviatano, Introduzione di C. Galli, Bologna, il Mulino, 2011.

-, Ex Captivitate Salus. Esperienze degli anni 1945-47 [1950], Con un saggio di F. Mercadante, trad. it. Milano, Adelphi, 1987.

-, La dittatura. Dalle origini dell'idea moderna di sovranità alla lotta di classe proletaria (1921), trad. it. Bari, Laterza, 1975.

Slongo, P., La forza della consuetudine. Costumi, costituzione, governo in Montaigne e Montesquieu, Milano, FrancoAngeli, 2020.

Spitz, J.-F., Bodin et la souveraineté, Paris, PUF, 1998.

Thomas, Y., "L'institution de la Majesté", Revue de Synthèse, 3-4, 1991, pp. 331-386.

-, Il valore delle cose (2002), a cura di M. Spanò, con un saggio di G. Agamben, Macerata, Quodlibet, 2015, pp. 74-75.

-, 'Res, choses et patrimoine. Note sur le rapport sujet-objet en droit romain", Archives de Philosophie du Droit, 25, 1980, pp. 413-426.

Vismara, G., "La disciplina giuridica del castello medievale (sec. VI-XIII)" (1972), ora in G. Vismara, Scritti di storia giuridica, vol. 4, Milano, Giuffrè, 1988, pp. 1-133.

Weber, M., "I tipi del potere", in M. Weber, Economia e società (1922), vol. I, trad. it. a cura di P. Rossi, Milano, Edizioni di Comunità, 1961, pp. 207-211.

-, Il metodo delle scienze storico-sociali (1922), a cura di P. Rossi, Torino, Einaudi, 1958.

Zarka, Y. C., a cura di, Jean Bodin. Nature, histoire, droit et politique, Paris, PUF, 1996. 